\title{
Assessment of Cataloging \\ Services in an Academic Library
}

\author{
Catherine Sassen \\ Principal Catalog Librarian
}

Kathryn Loafman

Head, Cataloging and Metadata Services

\author{
Rebecca Welch \\ Catalog Management Librarian
}

Texas Library Association Annual Conference

April 15, 2015 


\section{Outline}

\section{Background}

\section{Literature Review}

Methodology

\section{Results}

Implications for Strategic Planning

Tips for Survey Administration 
Why did we want to do this study?

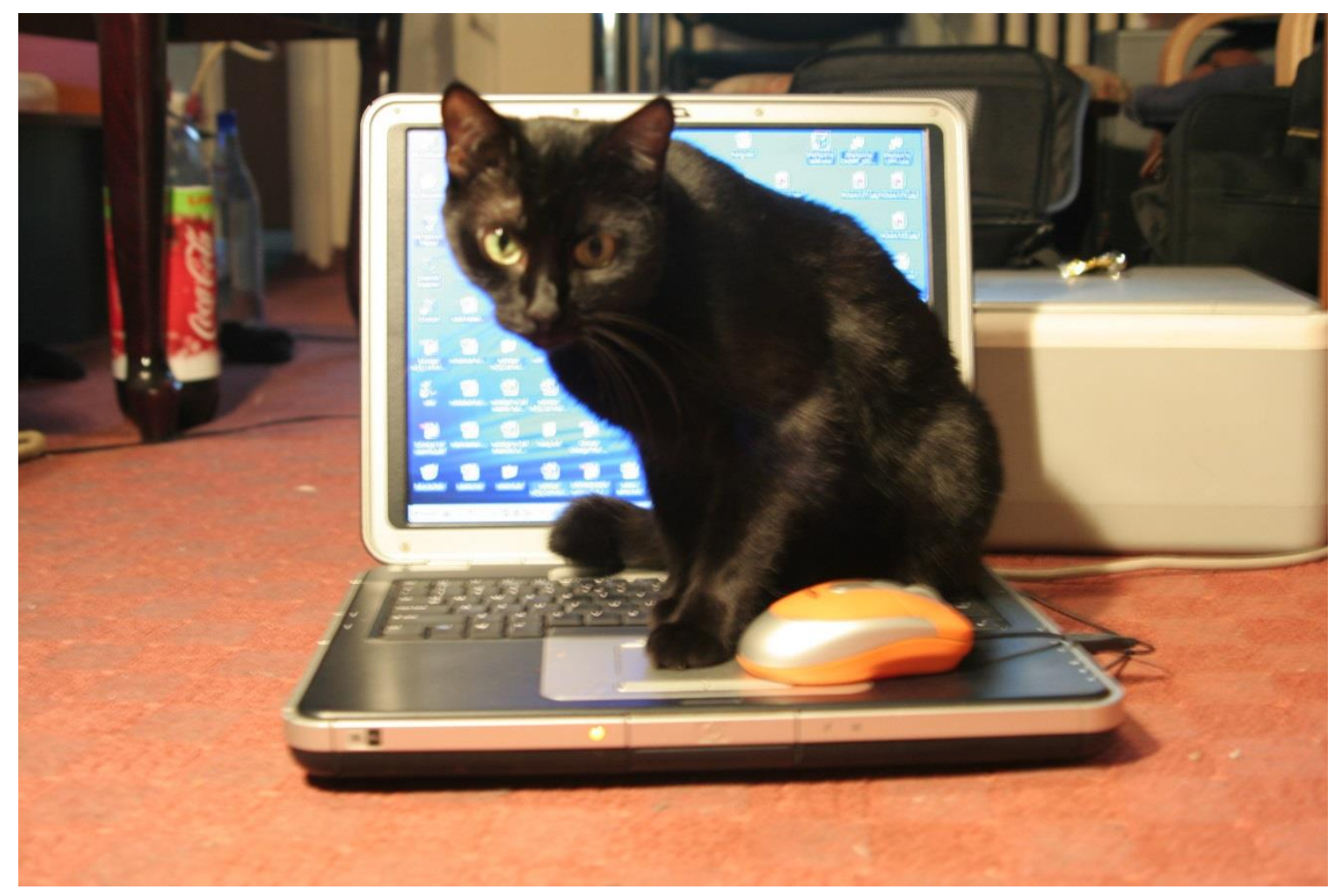




\section{Background}

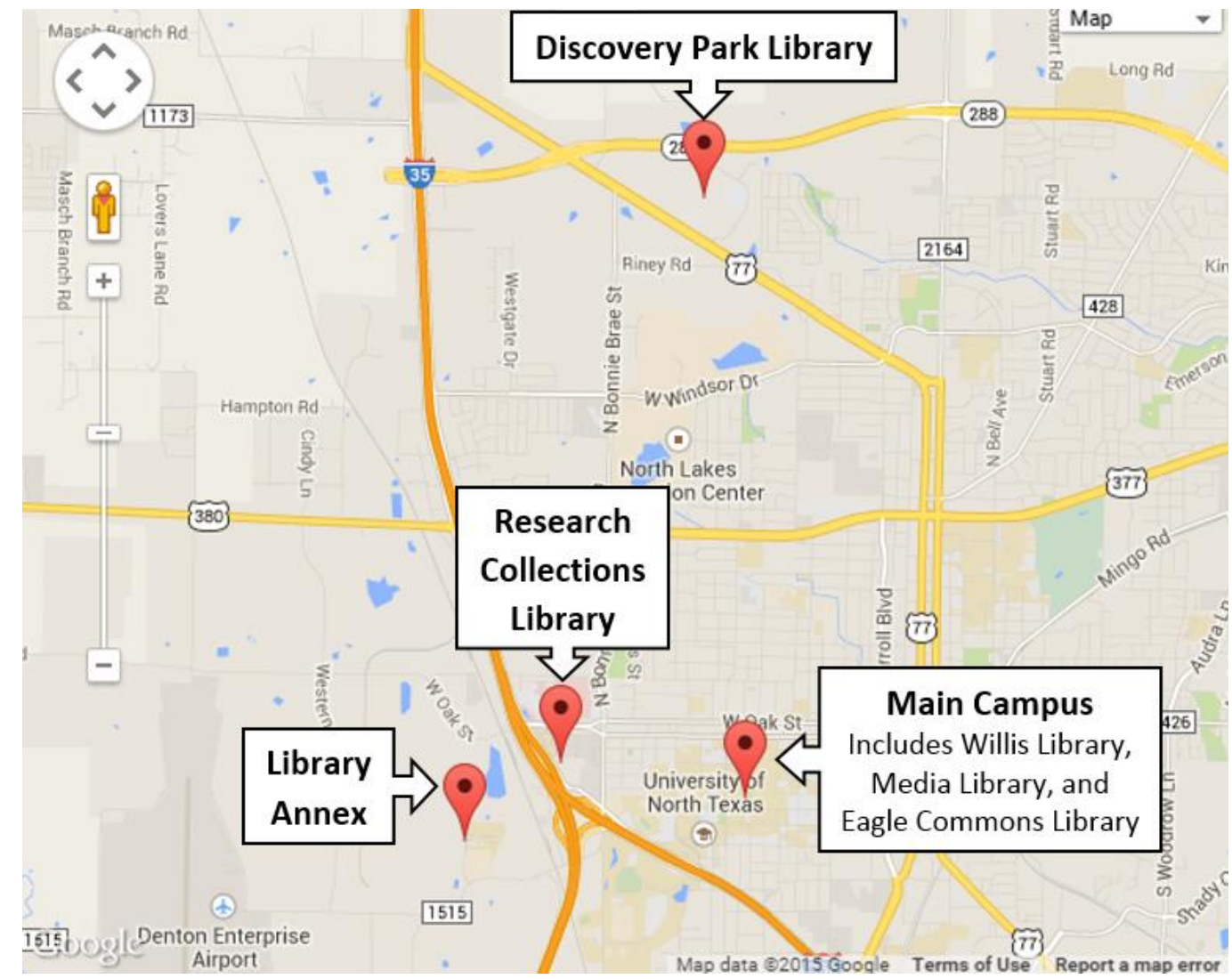

- Cataloging and Metadata Services and Collection Development Departments located off campus

- Public Services and Special Libraries Divisions housed in four other buildings 


\section{Change in record source}

Up to 2008

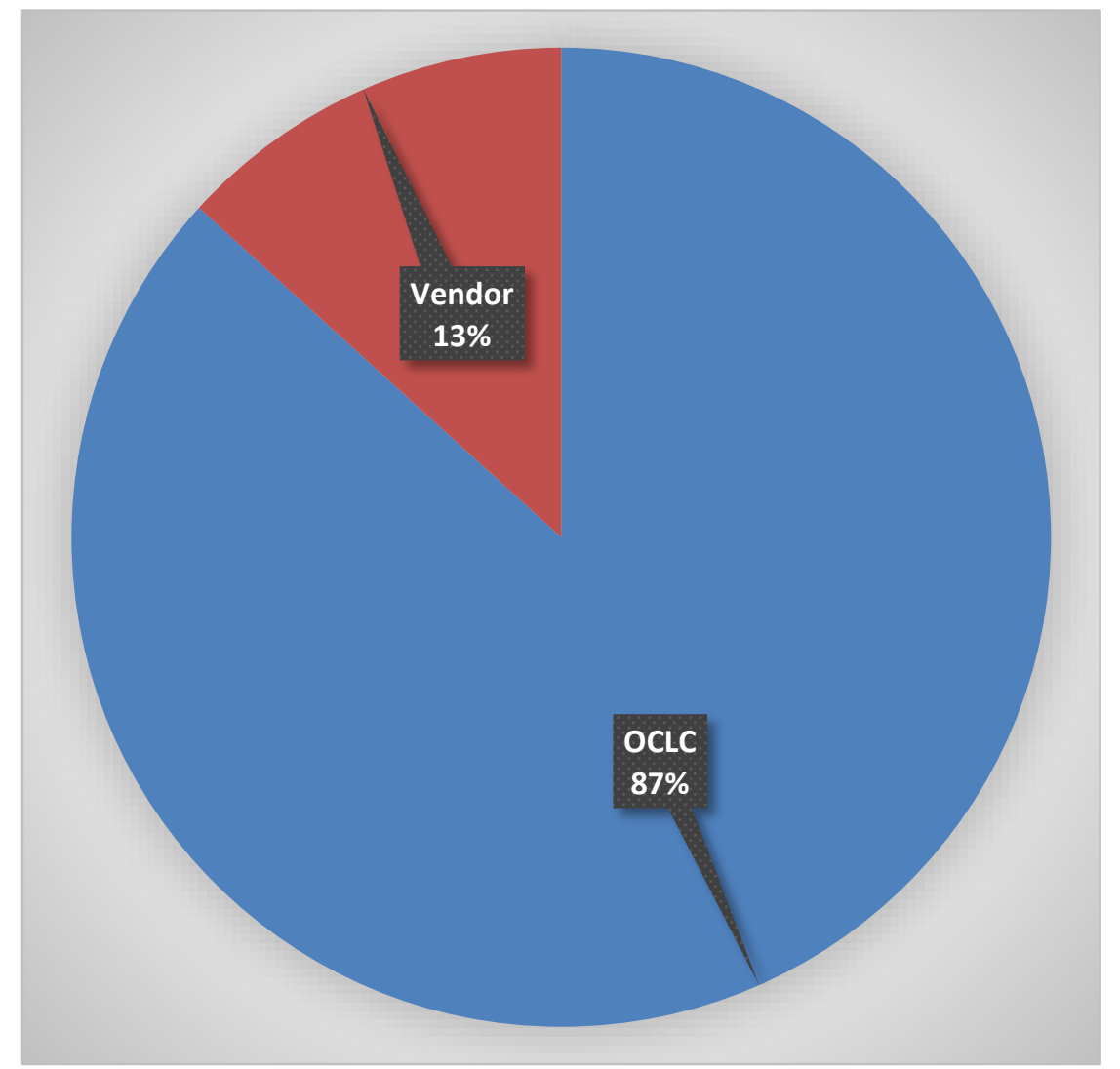

2008 to Present

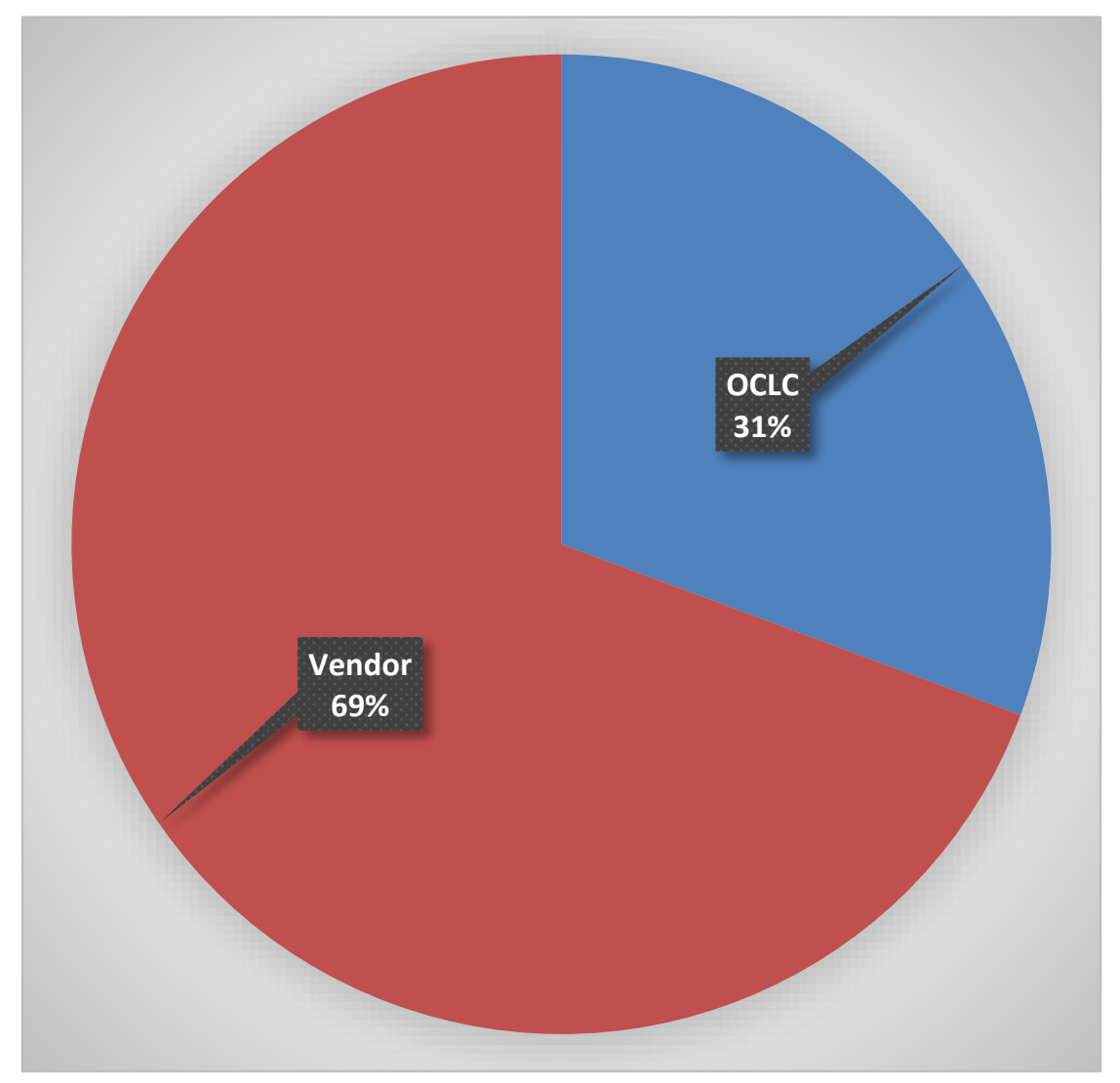




\section{Research questions}

- Importance

- Satisfaction

- Are we on the same page?

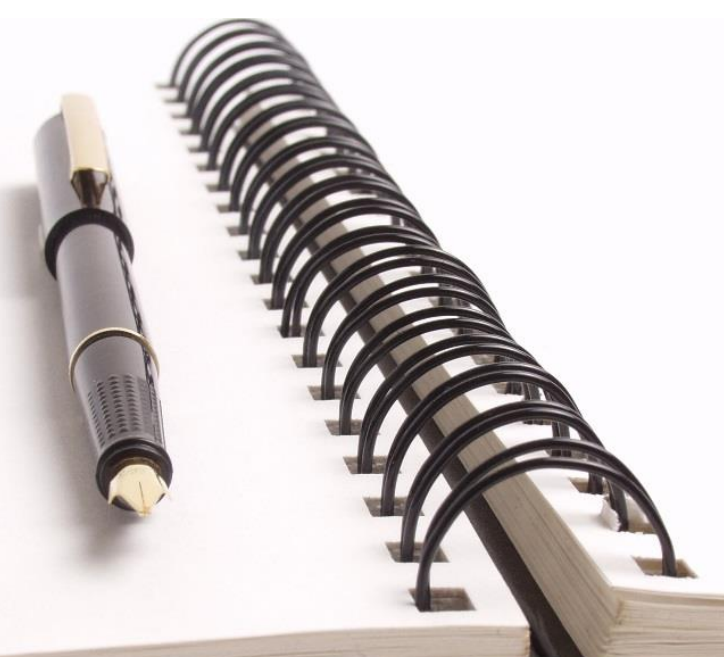




\section{Literature review}

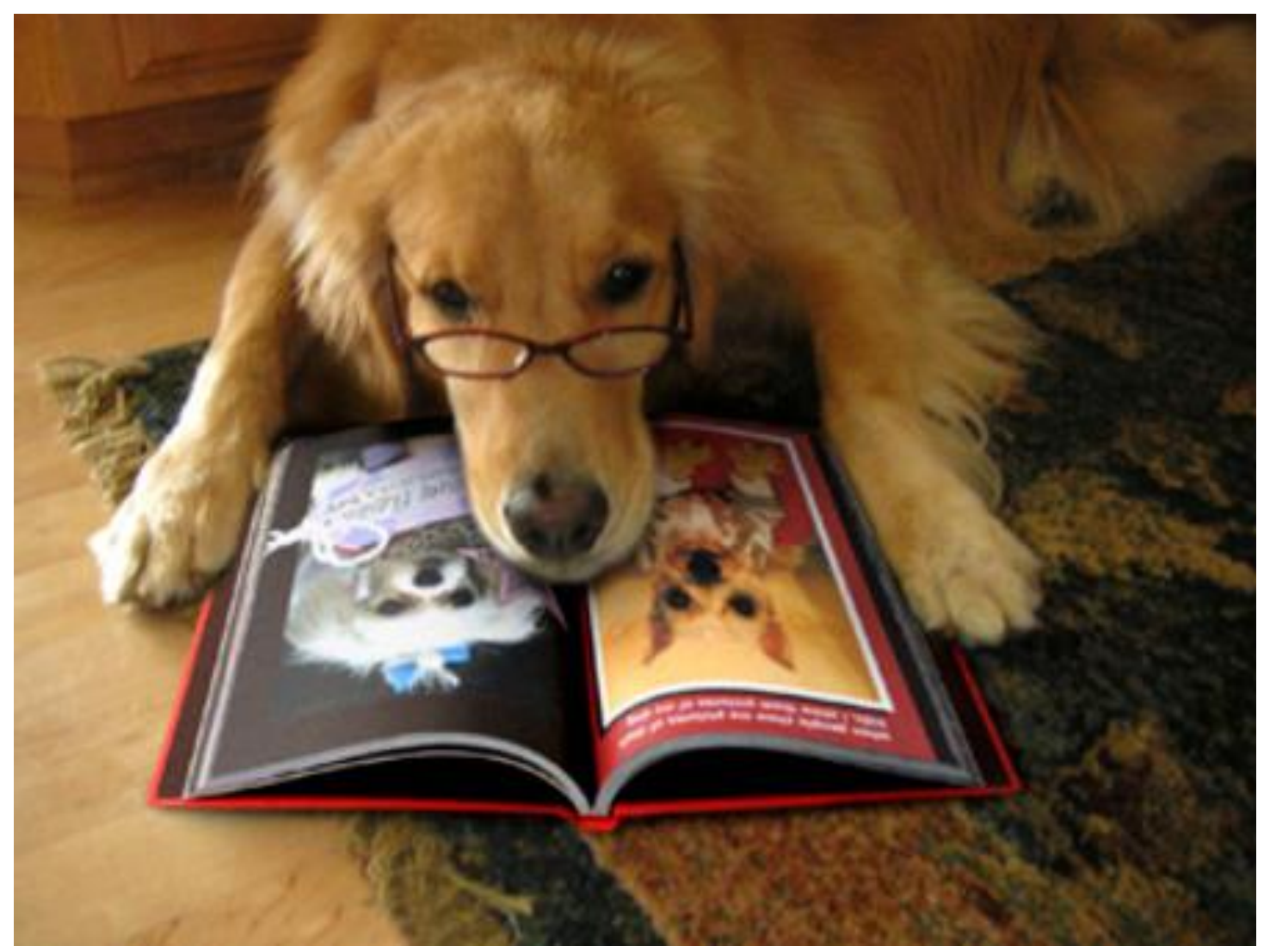




\section{Literature review}

Herrera, G., Cheng, D. T., Leslie, J., \& Harry, T.

(2006). Technical services cataloging and database maintenance assessment. Technical Services Quarterly 23(3), 51-72. 


\section{Literature review - cont.}

- Findings:

- High importance and satisfaction levels

- Opinions differed on top priorities

- The survey brought issues to the surface

- Staff education opportunities

- Increased awareness of services 


\section{Methodology}

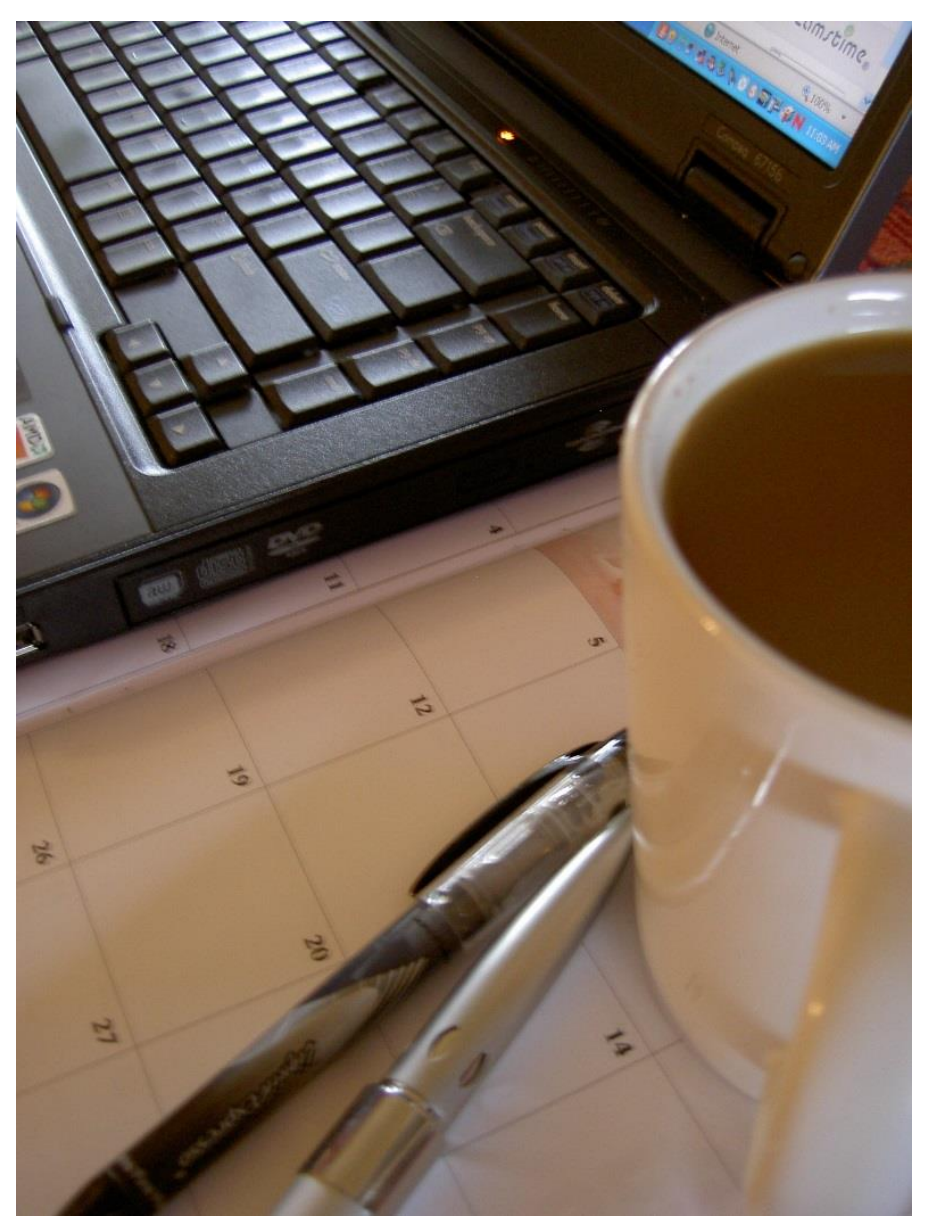




\section{Methodology}

- One online survey for Public Services

Division and Special Libraries Division

- A separate copy of the survey for Cataloging and Metadata Services to assess discrepancies

- Administered for three weeks in fall 2014 


\section{Response rate}

- Public Services, Special Libraries [PS]

-36 of 74 responded (48\%)

- Cataloging and Metadata Services [CMS]

-7 out of 8 responded ( $87 \%$ ) 


\section{Scale: Importance}

Q2. How important are the following cataloging services provided by the Cataloging and Metadata Services Department?

$\begin{array}{llll}\text { No } & \text { Low } & \text { Moderate } & \text { High } \\ \text { importance } & \text { importance } & \text { importance } & \text { importance }\end{array}$

Cataloging purchased print books

0

O

O
$\mathrm{O}$

O

O

0
$\mathrm{O}$

0

O 


\section{Scale: Satisfaction}

Q3. How satisfied are you with the following cataloging services provided by the Cataloging and Metadata Services Department?

\begin{tabular}{l|cccc} 
& Dissatisfied & $\begin{array}{c}\text { Somewhat } \\
\text { dissatisfied }\end{array}$ & $\begin{array}{c}\text { Somewhat } \\
\text { satisfied }\end{array}$ & Satisfied \\
\hline $\begin{array}{l}\text { Cataloging purchased } \\
\text { print books }\end{array}$ & 0 & 0 & 0 & 0 \\
Cataloging gift books & 0 & 0 & 0 & 0 \\
Cataloging e-books & 0 & 0 & 0 & 0
\end{tabular}




\section{Scoring}

Importance scale

$1=$ No importance

2=Low importance

3=Moderate importance

4=High importance
Satisfaction scale

1=Dissatisfied

$2=$ Somewhat dissatisfied

$3=$ Somewhat satisfied

4=Satisfied 
Results

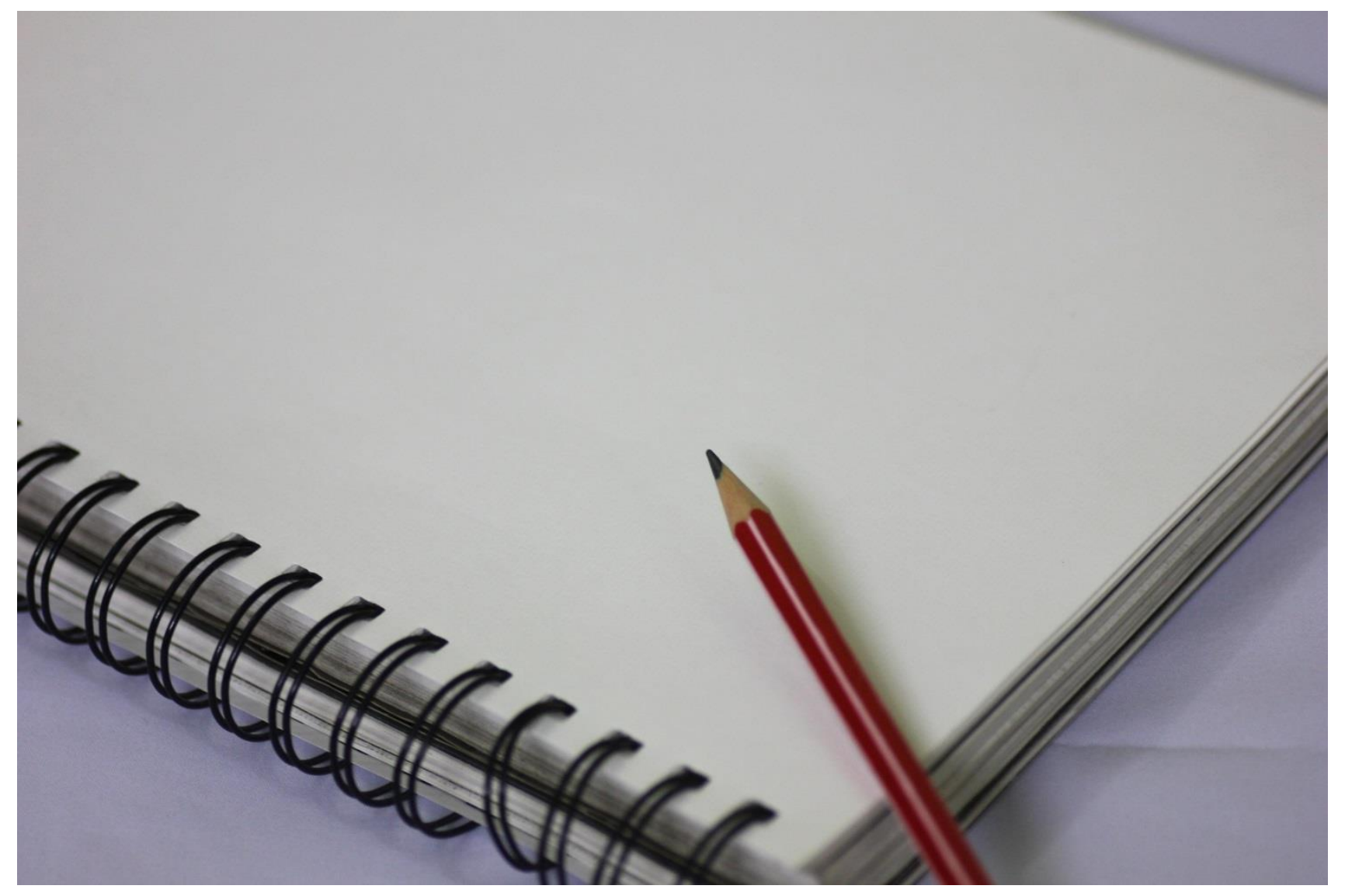




\section{Overall results}

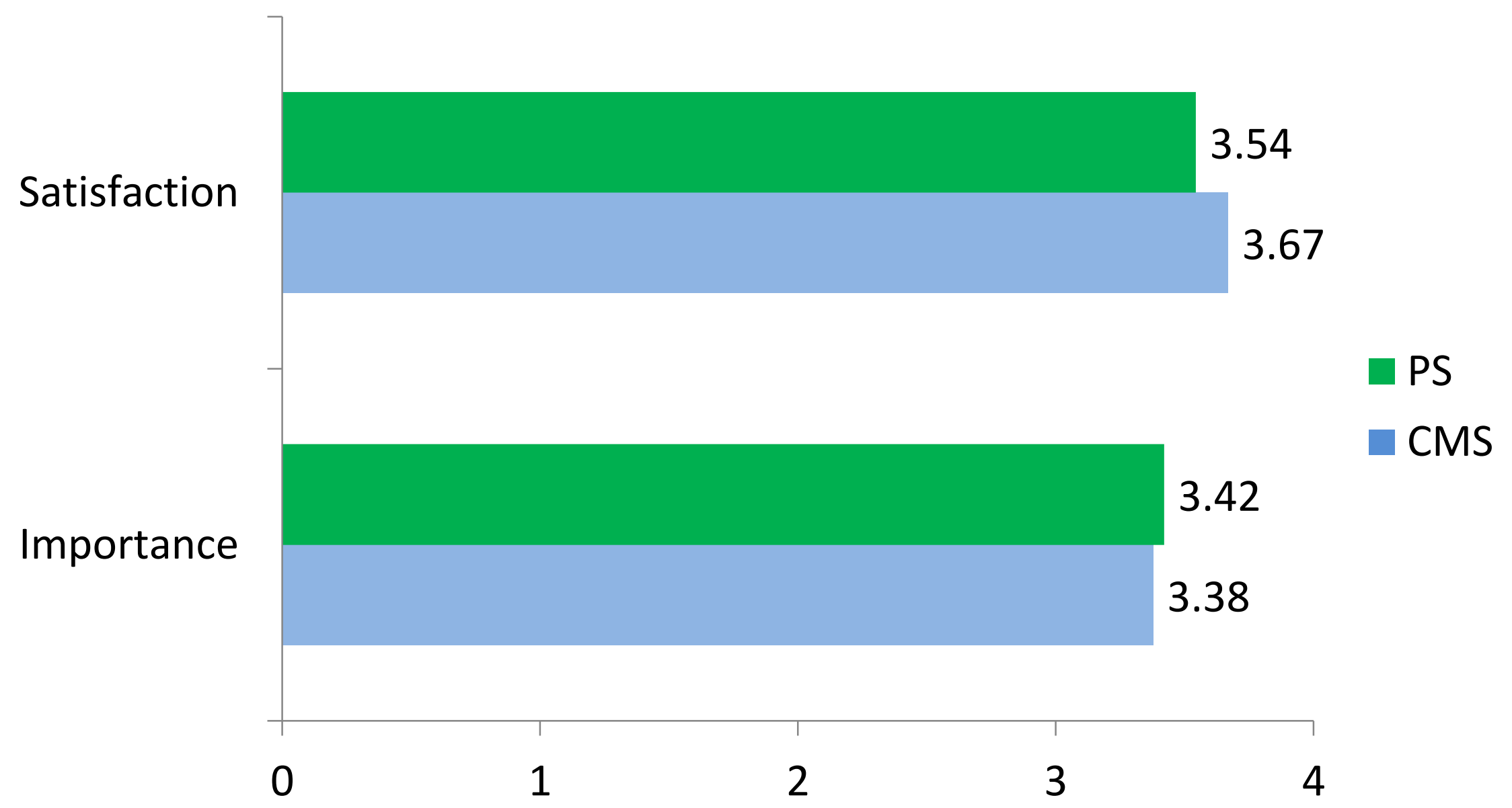




\section{What were the lowest scores?}

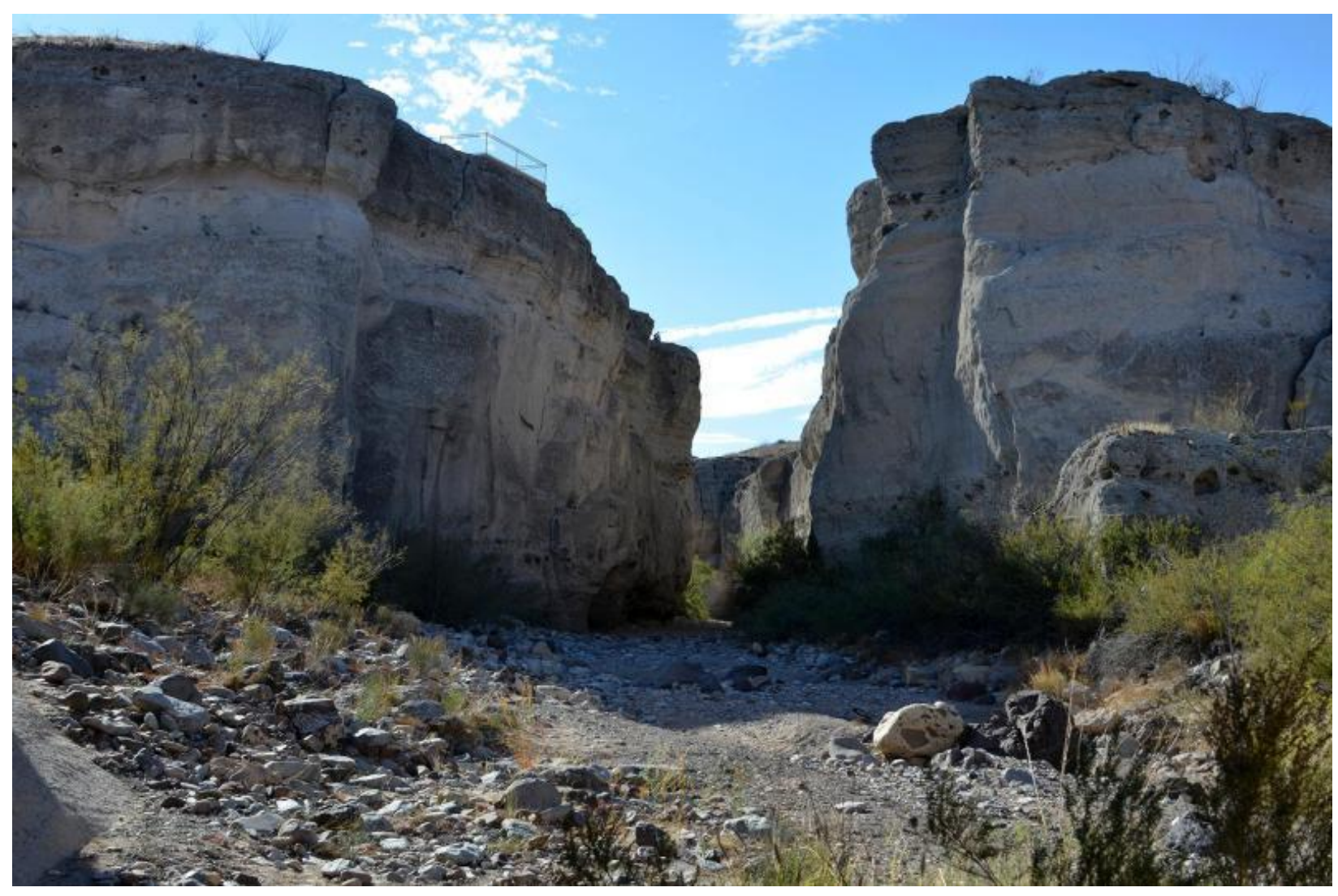




\section{Cataloging \\ foreign language gift books}

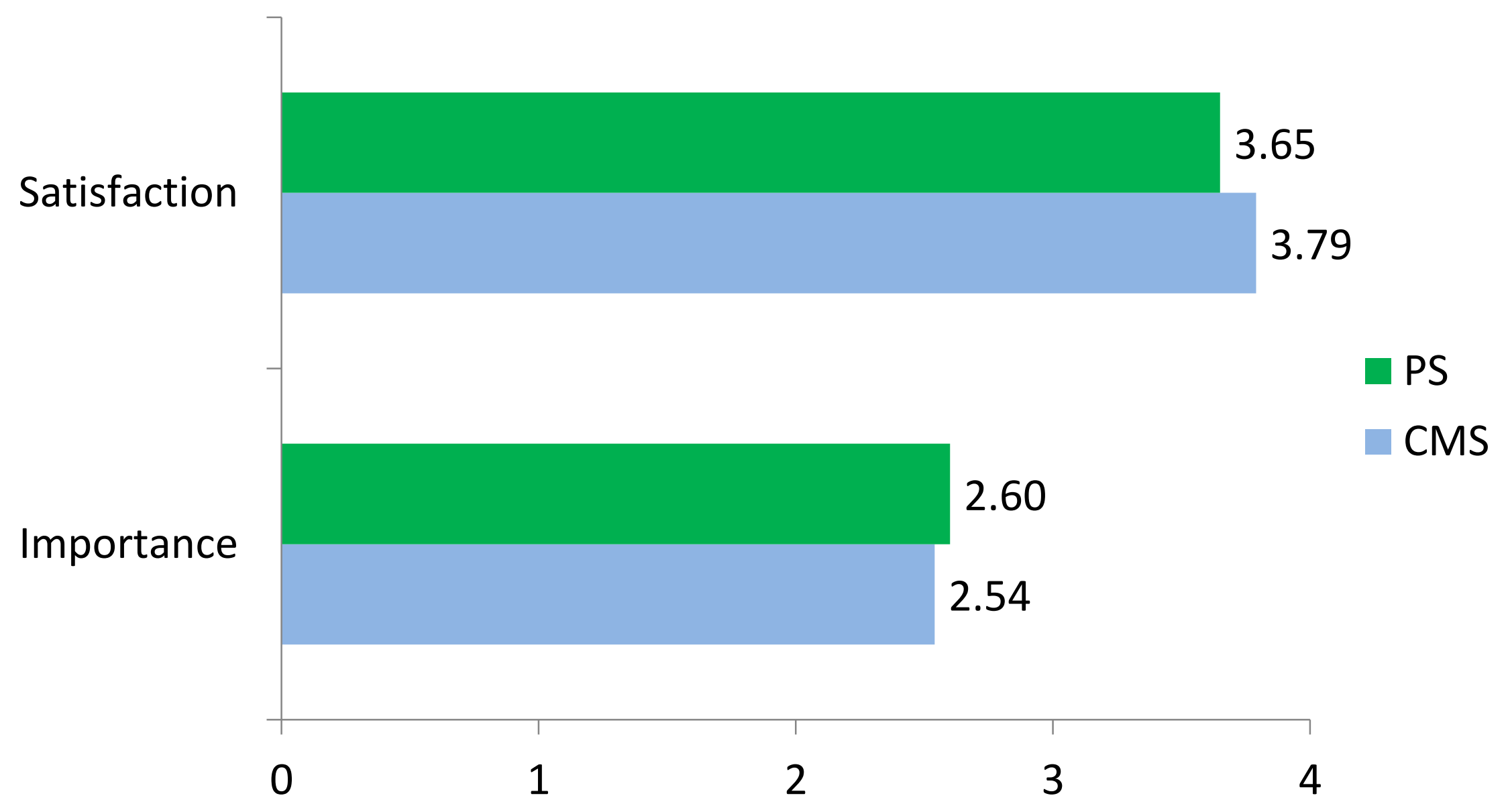




\section{Subject headings are consistently used and cross references are provided}

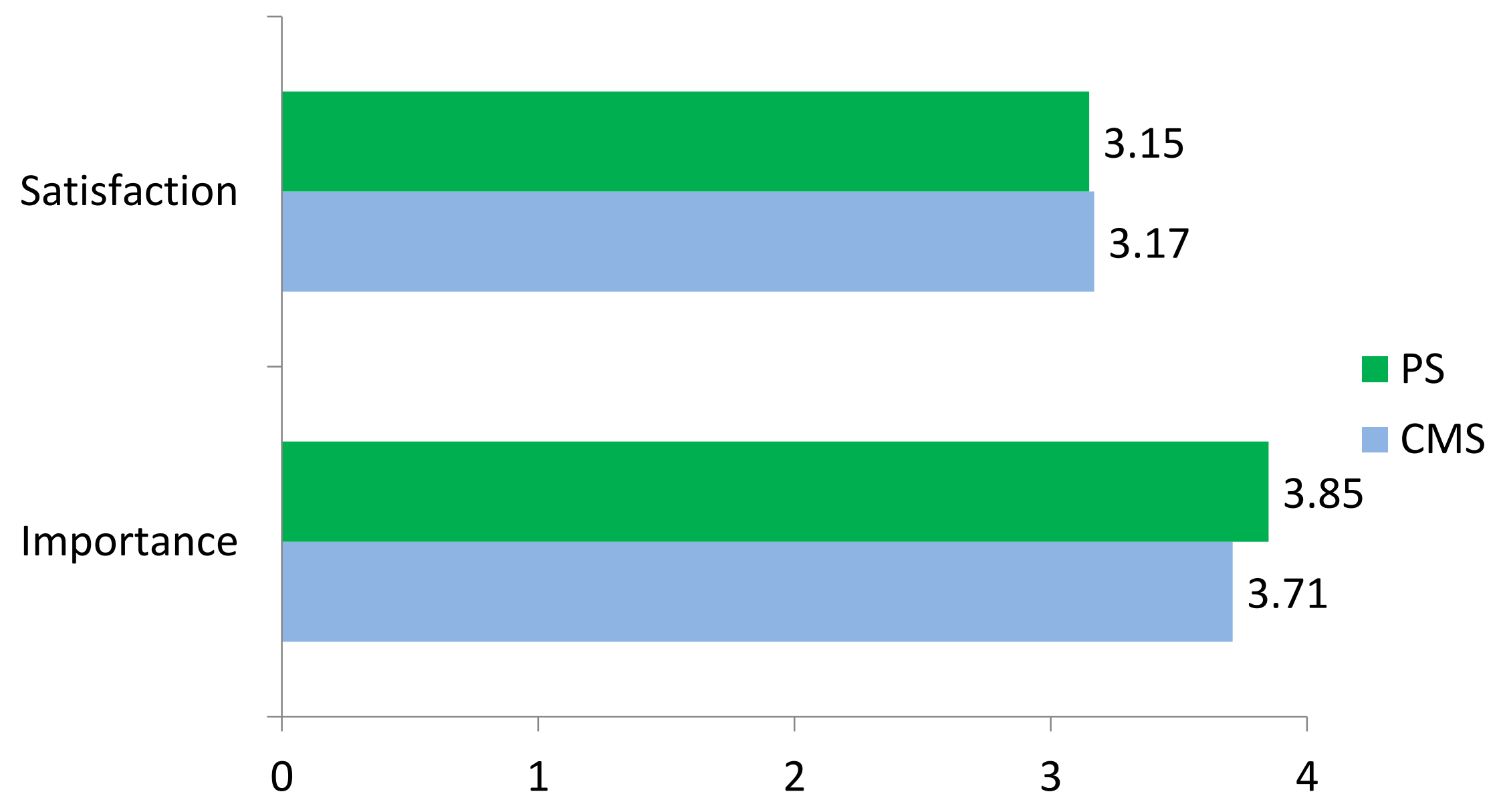




\section{Updating older minimal level periodical records}

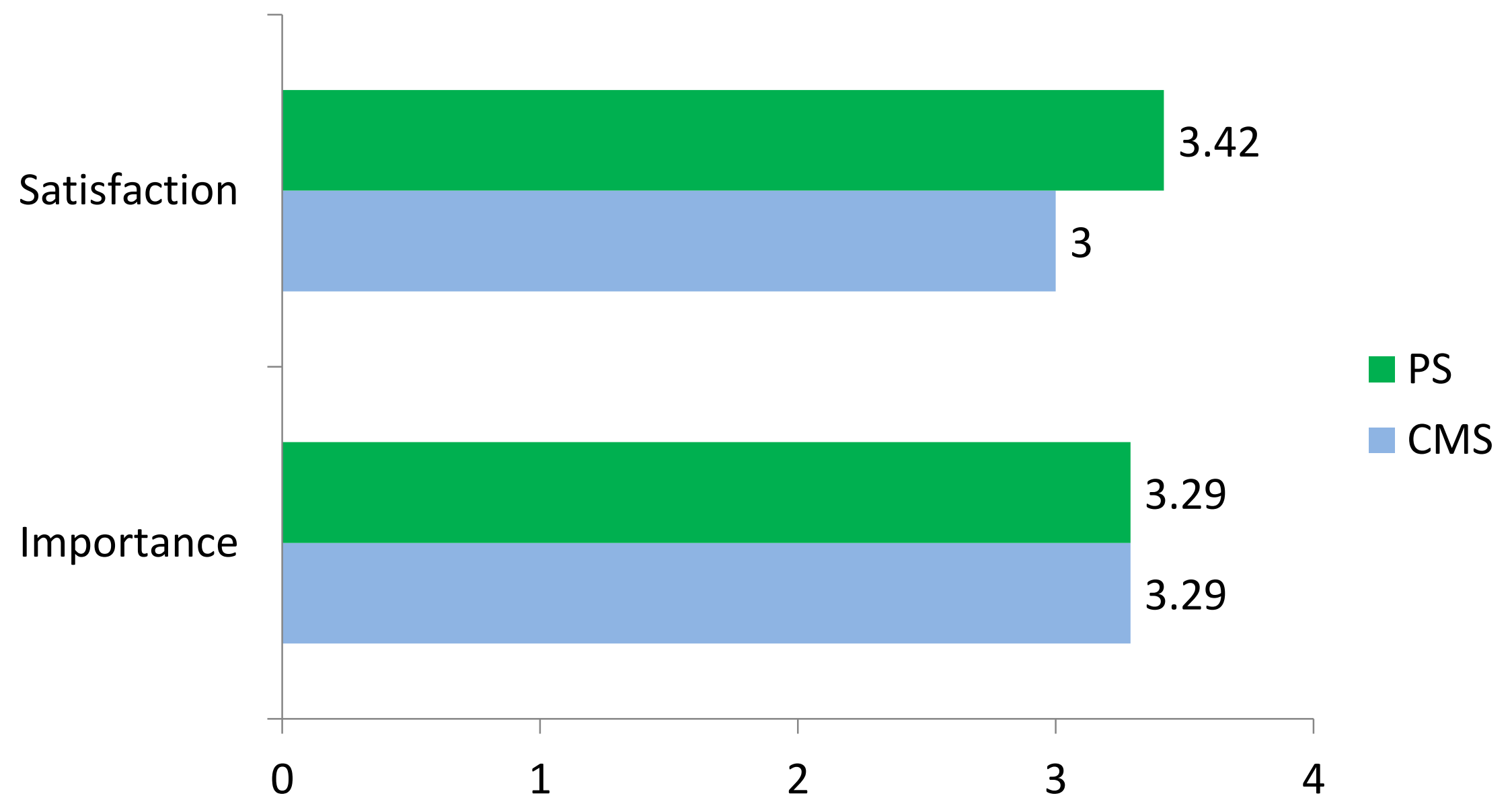




\section{What were the highest scores?}

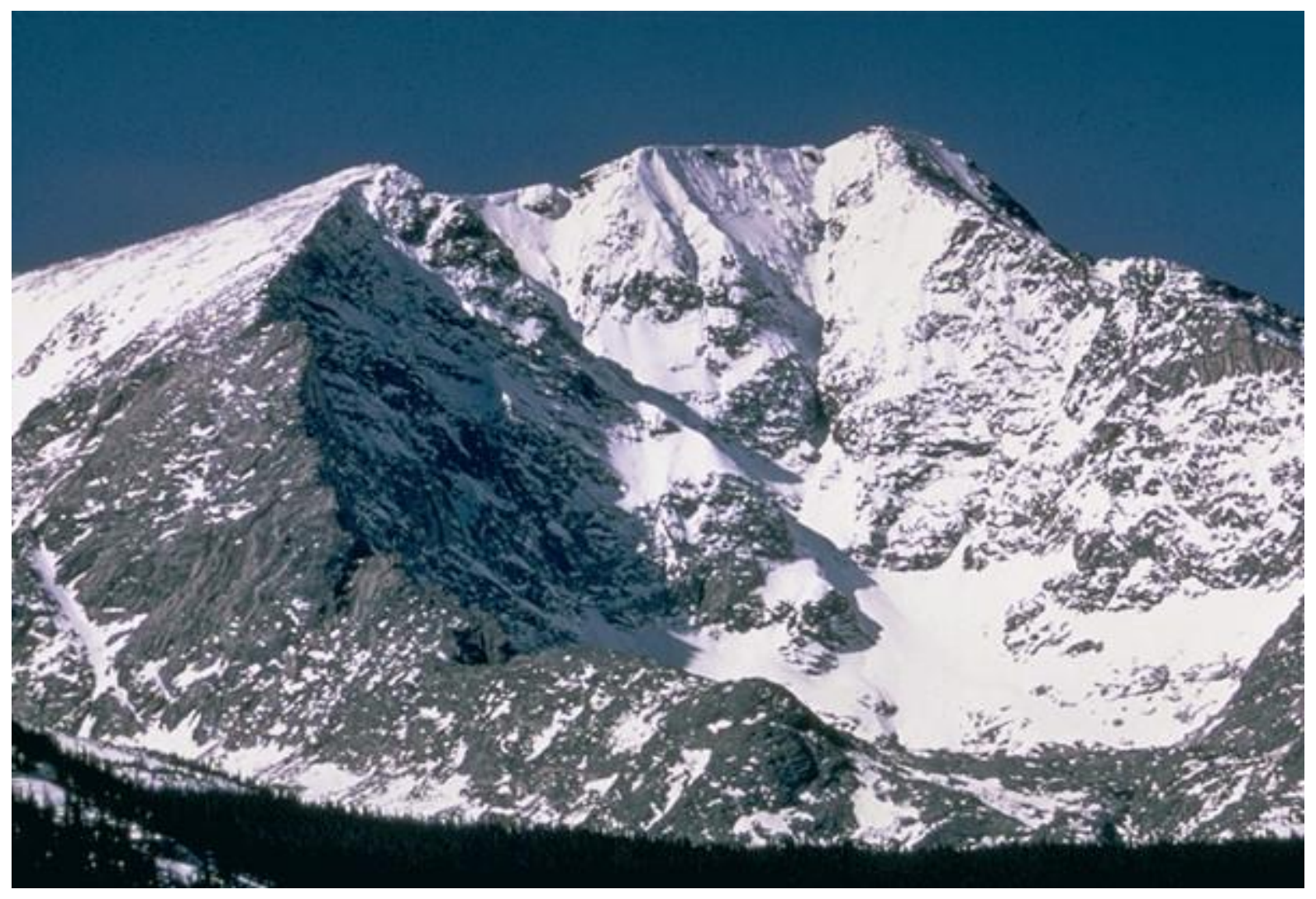




\section{Cataloging purchased print books}

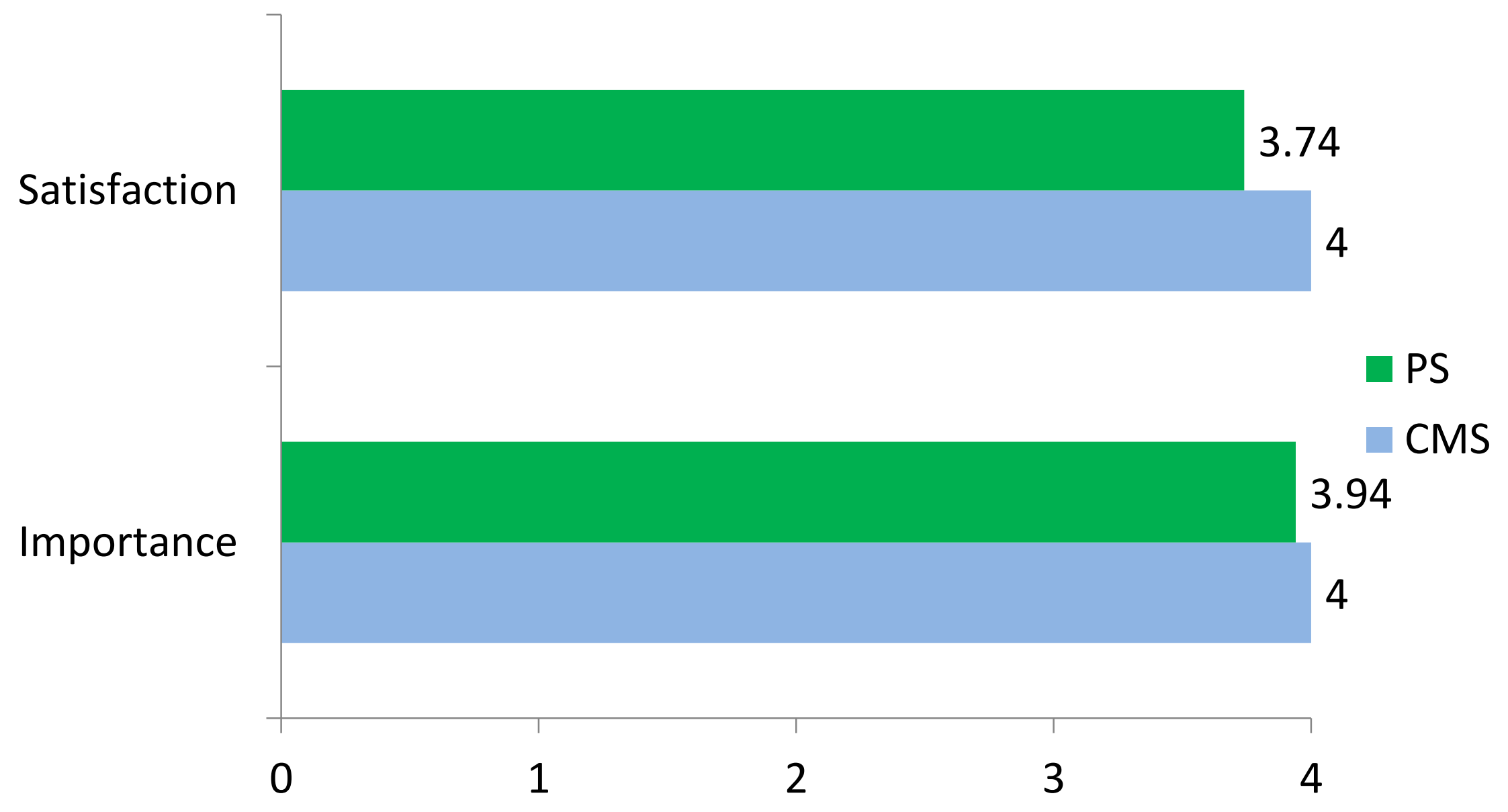




\section{Cataloging rush items}

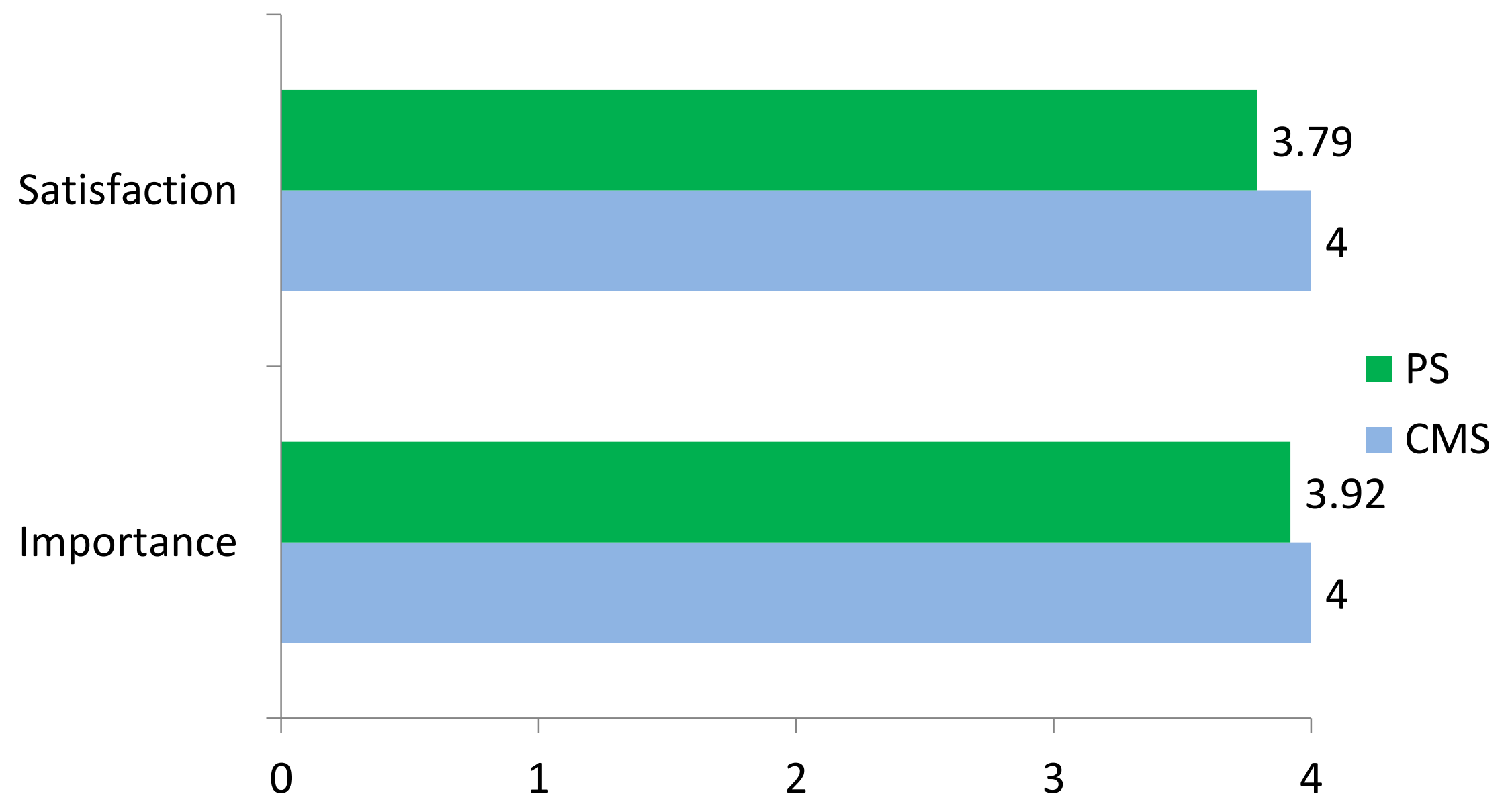




\section{What were the areas of greatest disagreement?}

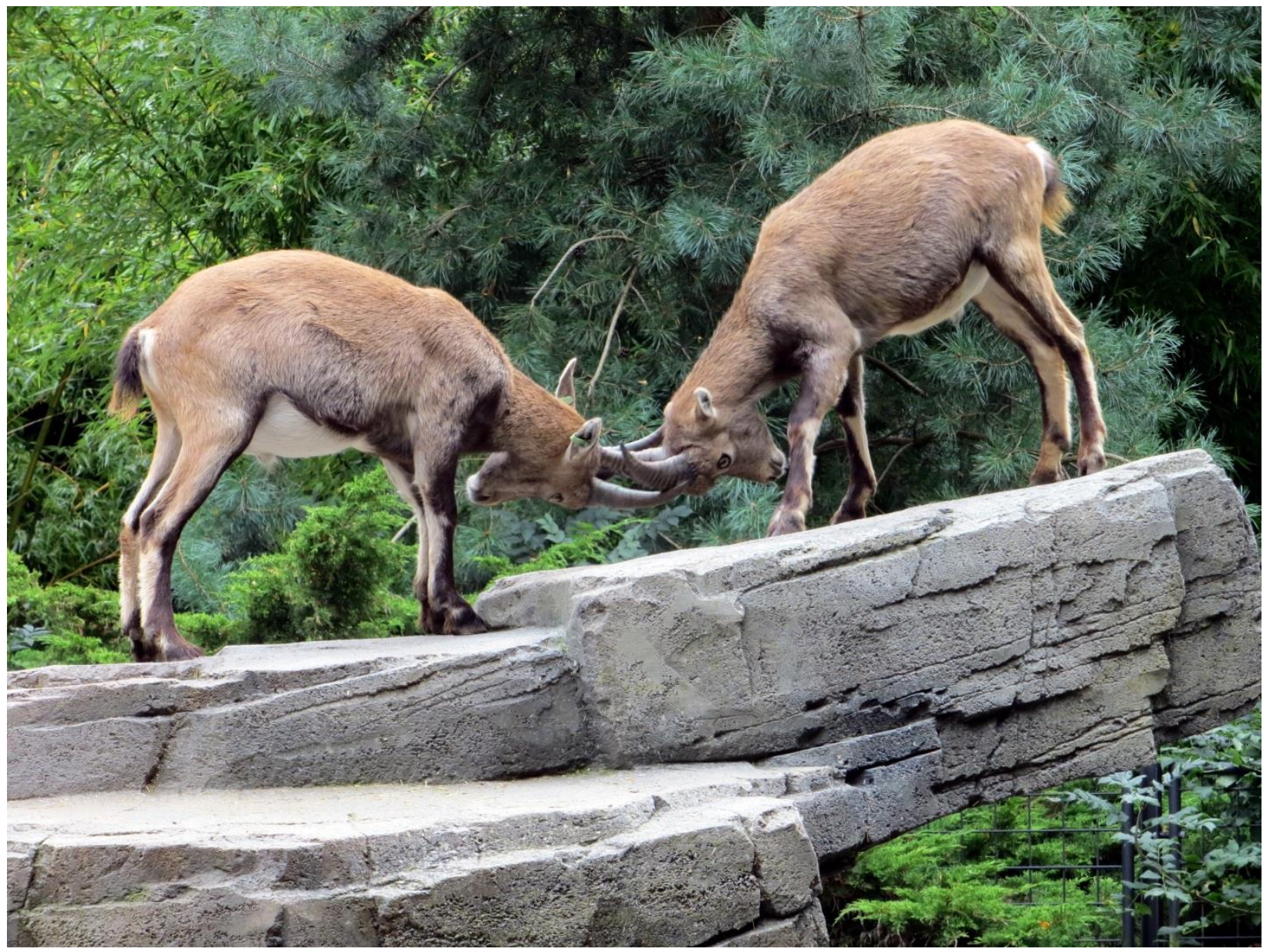




\section{Differences in scores overall}

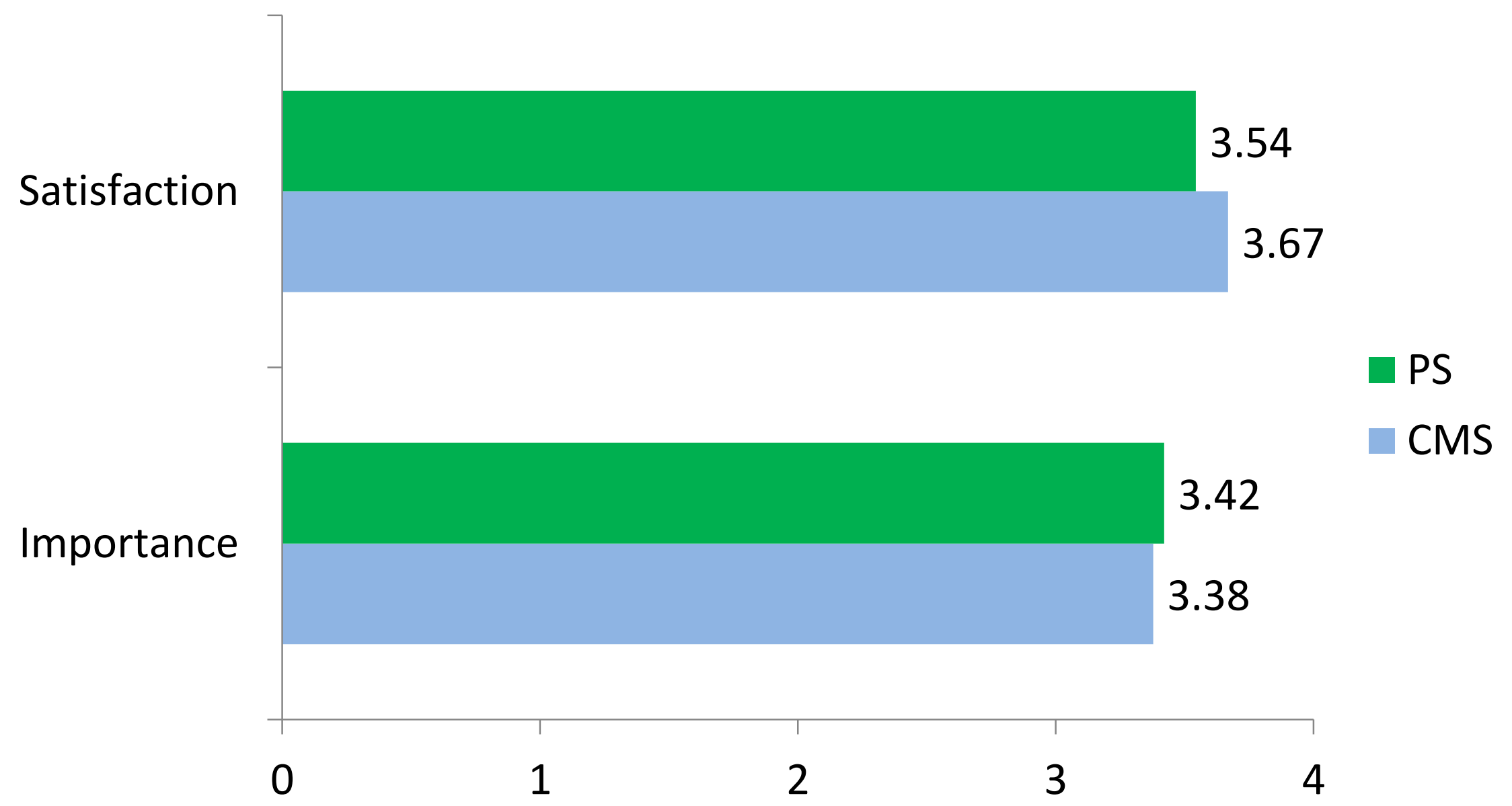




\section{Adding birth/death dates to personal name headings in the online catalog}

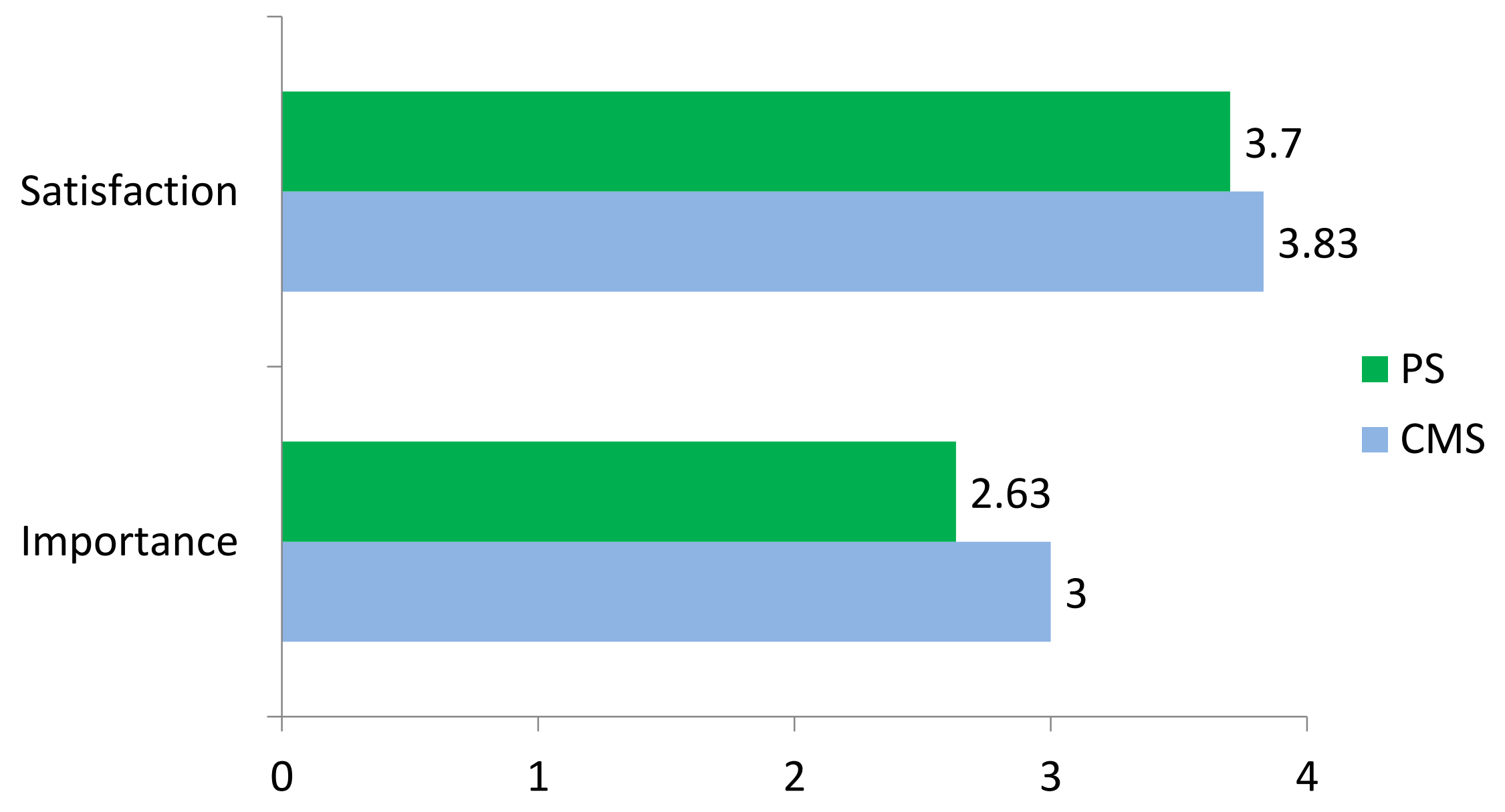




\section{All works by the same author are grouped together in the catalog}

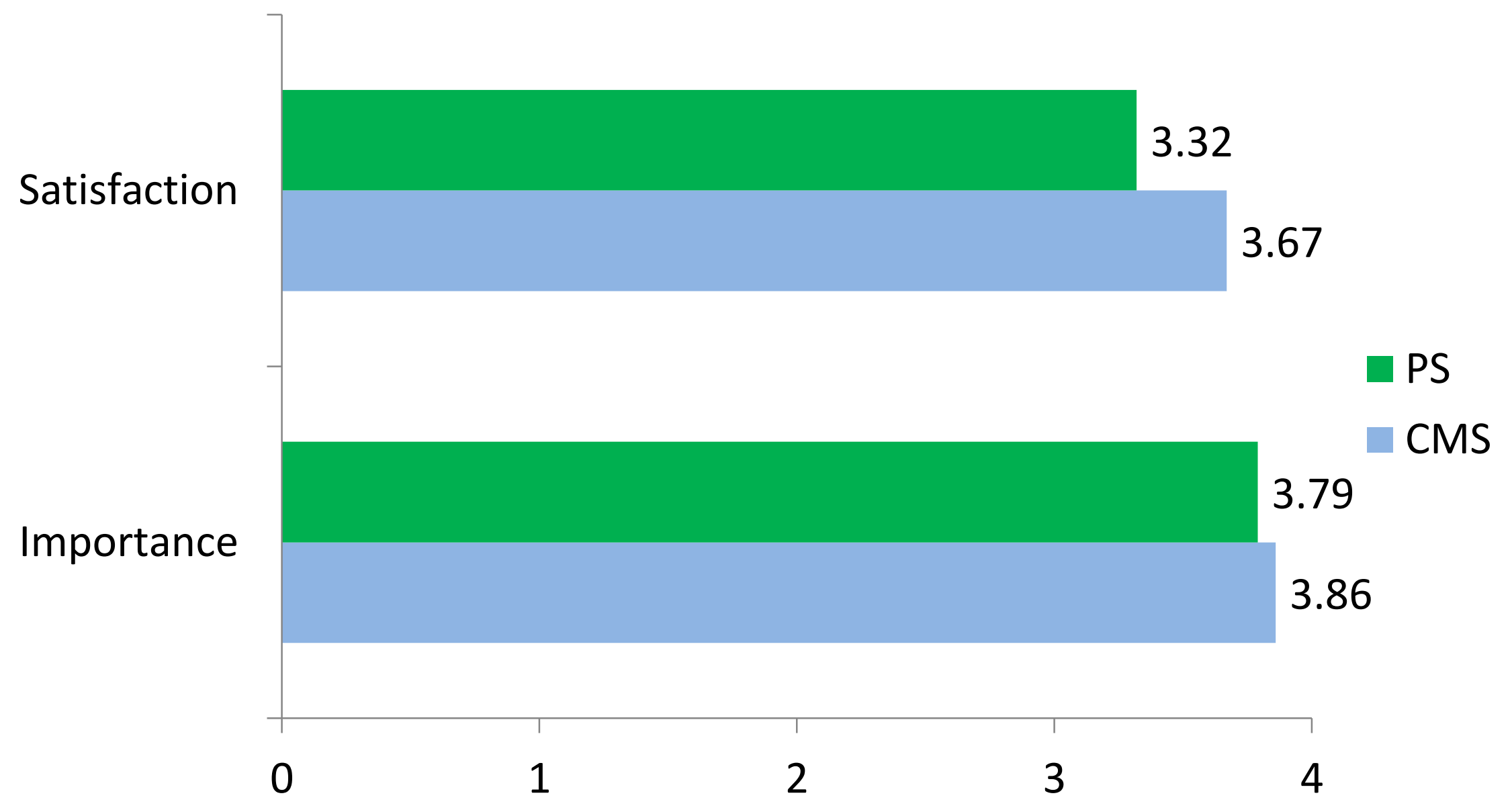




\section{Cataloging gift books}

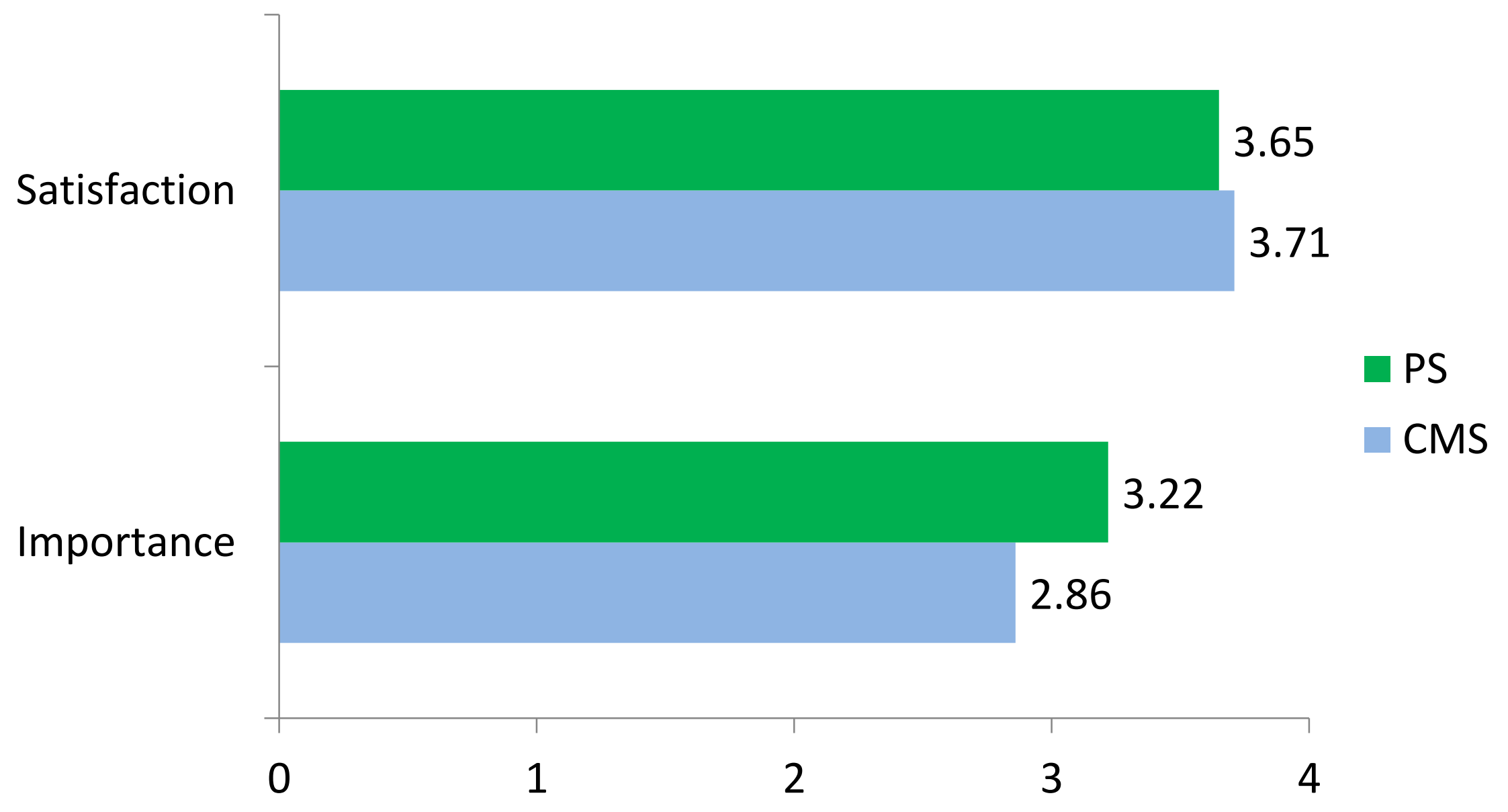




\section{Creating records for distinctive titles in monographic series}

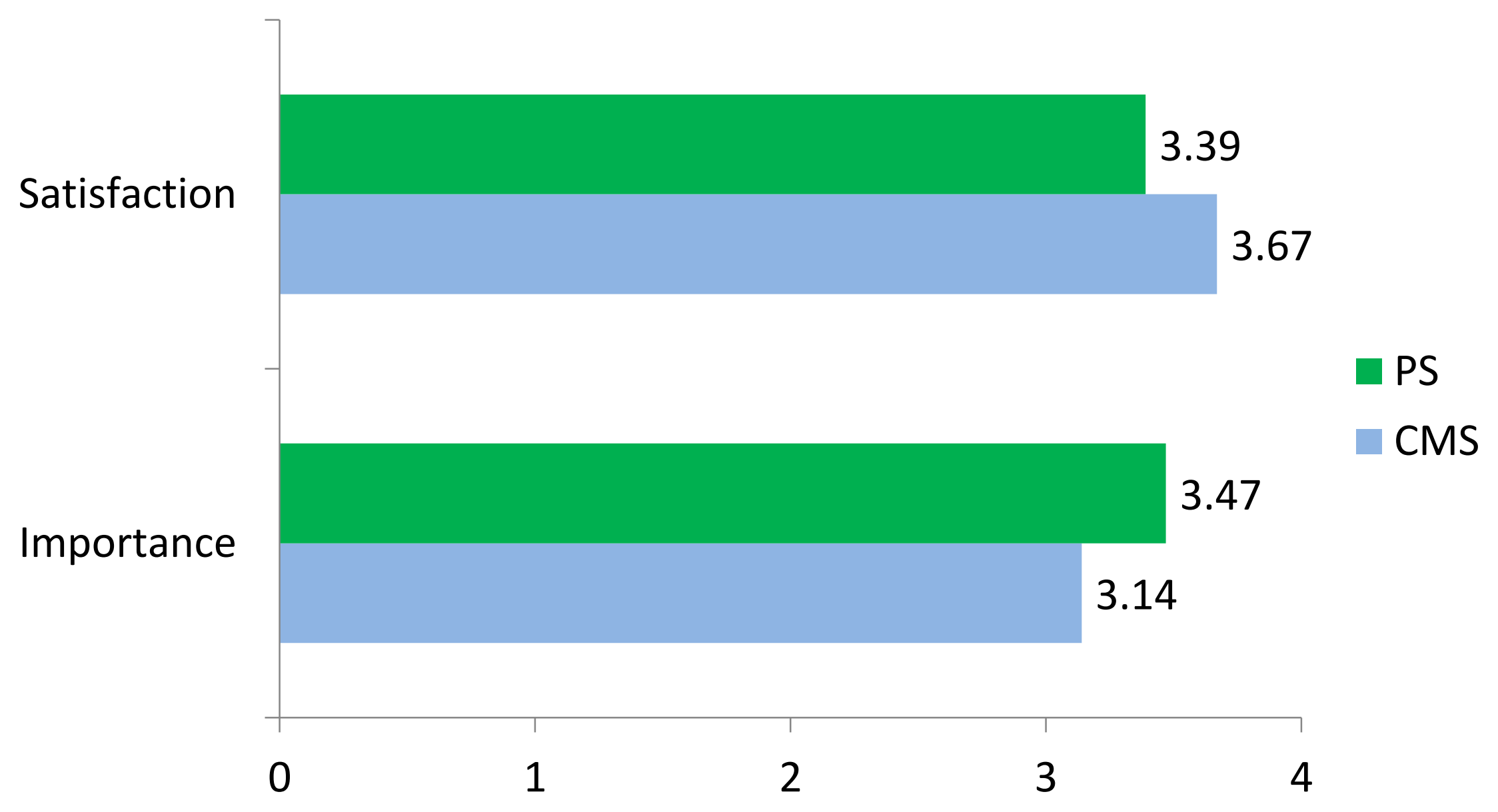




\section{Resolving problems with call numbers}

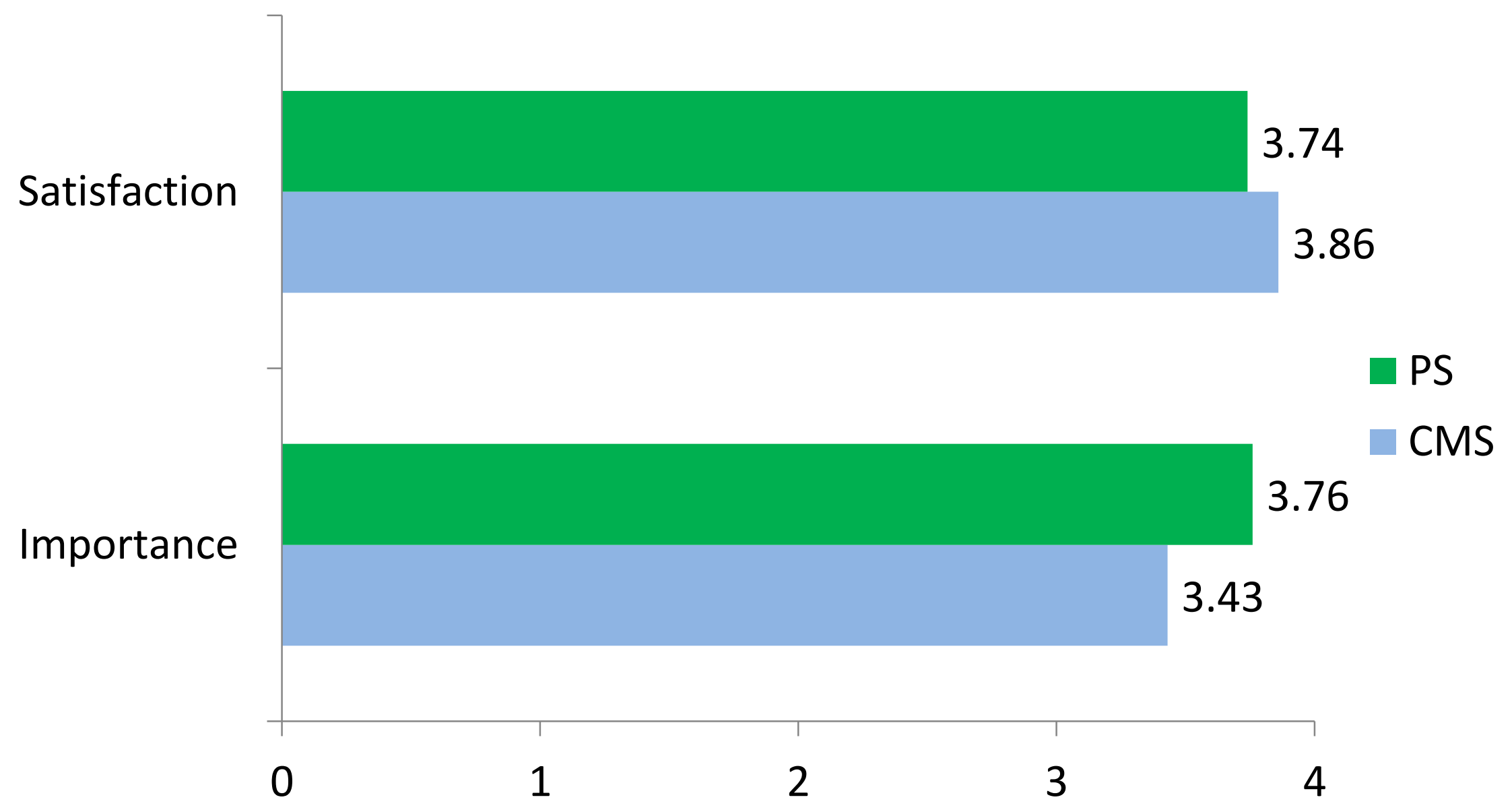




\section{What were the areas of greatest agreement?}

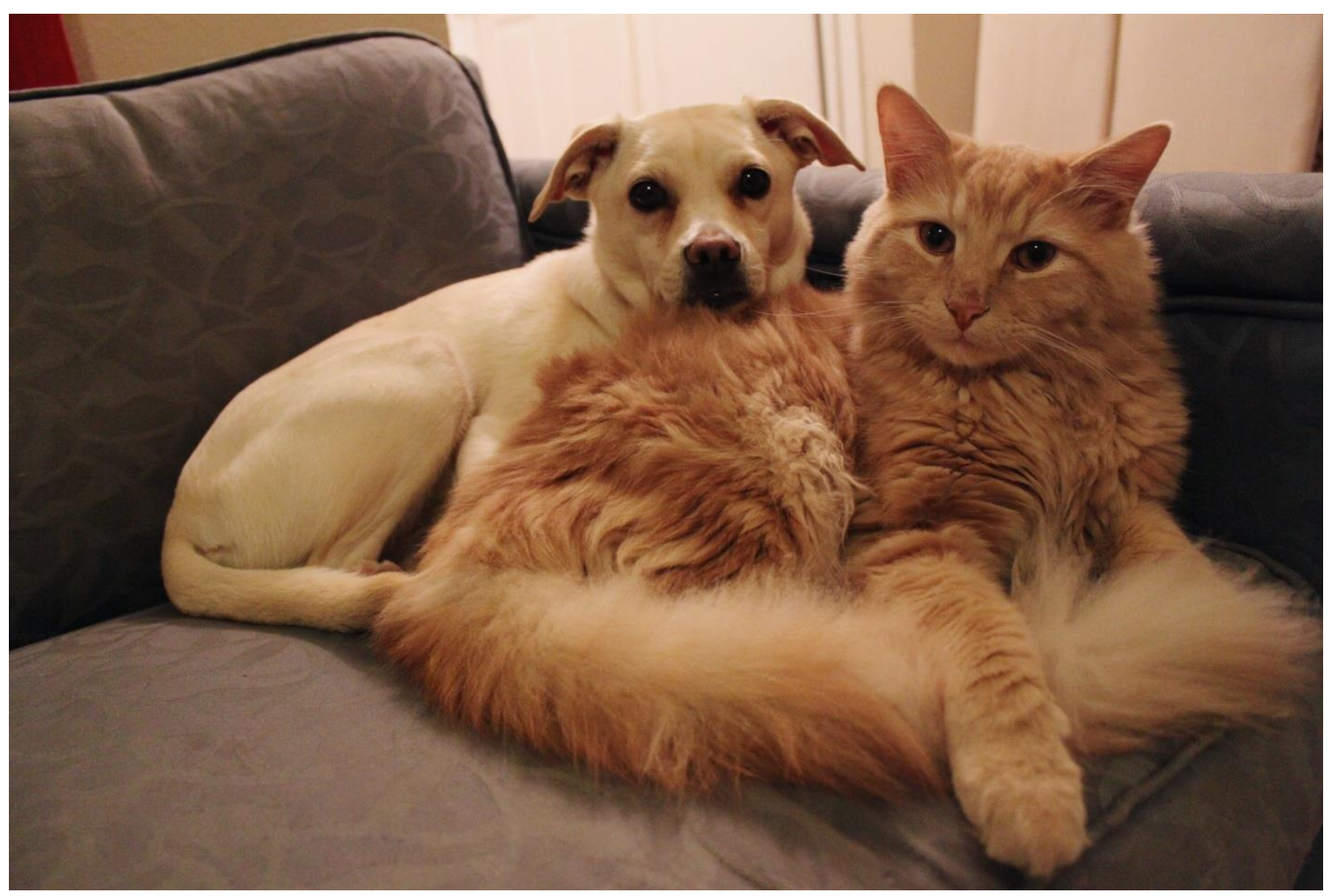




\section{Editing records in our online catalog for lost or missing materials}

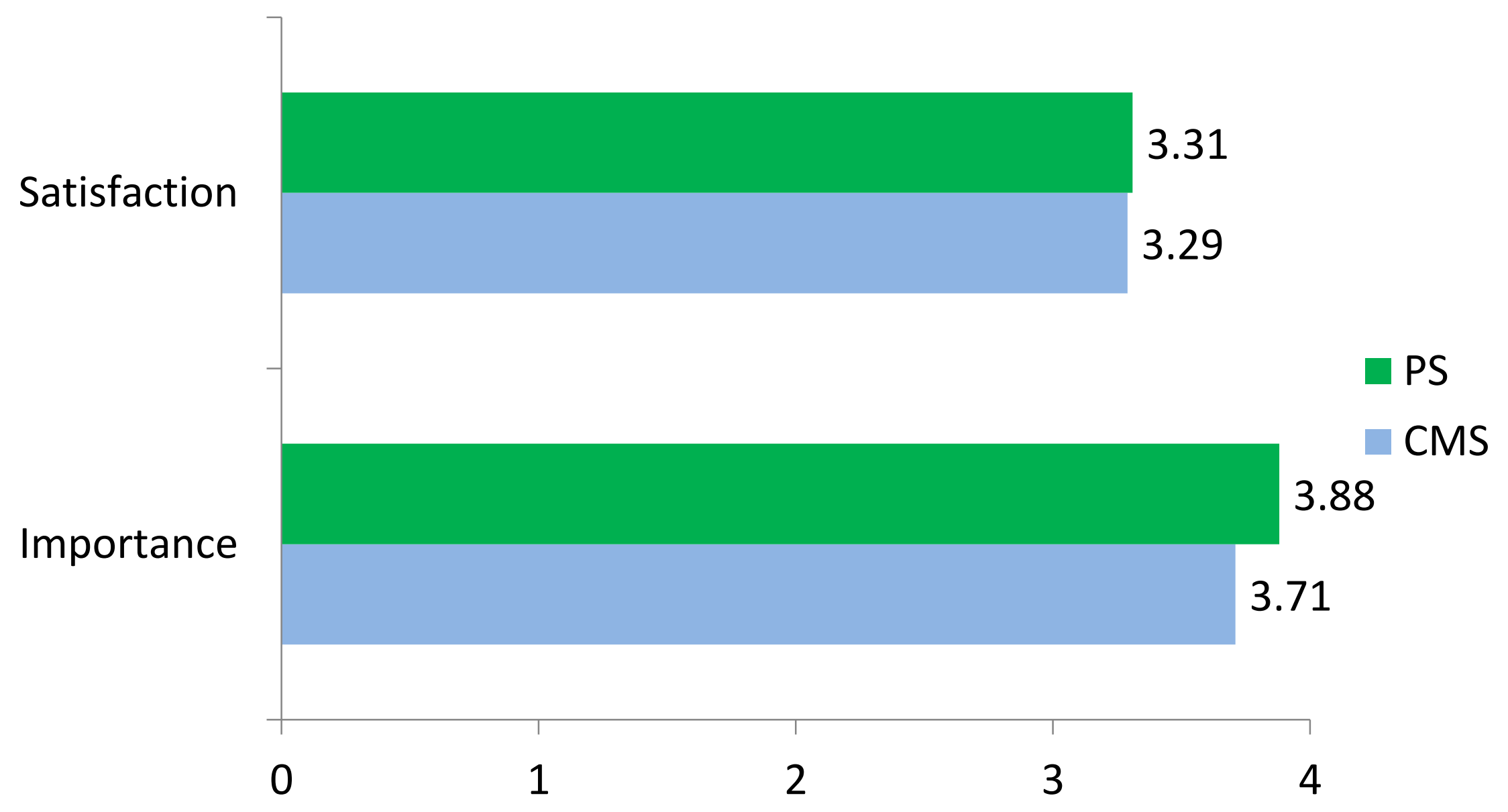




\section{Open-ended question}




\section{Open-ended question}

Please add any comments that you would like to make about the services of the Cataloging and Metadata Services Department.

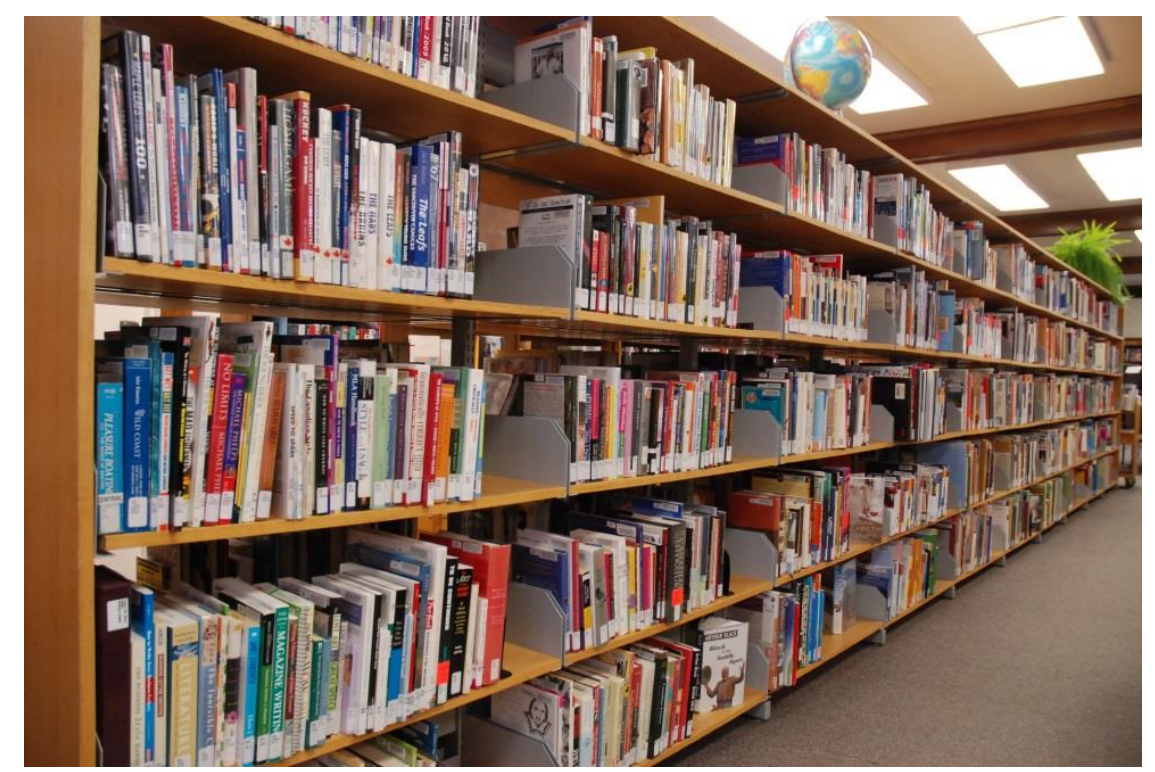


Responses to open-ended question

- Broken links

- Quality of microform records

- Display of volumes held 


\section{Responses to open-ended question}

"You guys rock!"

"Thanks for being so responsive when we email you guys with questions."

"Thank you for all that you do and keep up the good work!" 


\section{Strategic planning}

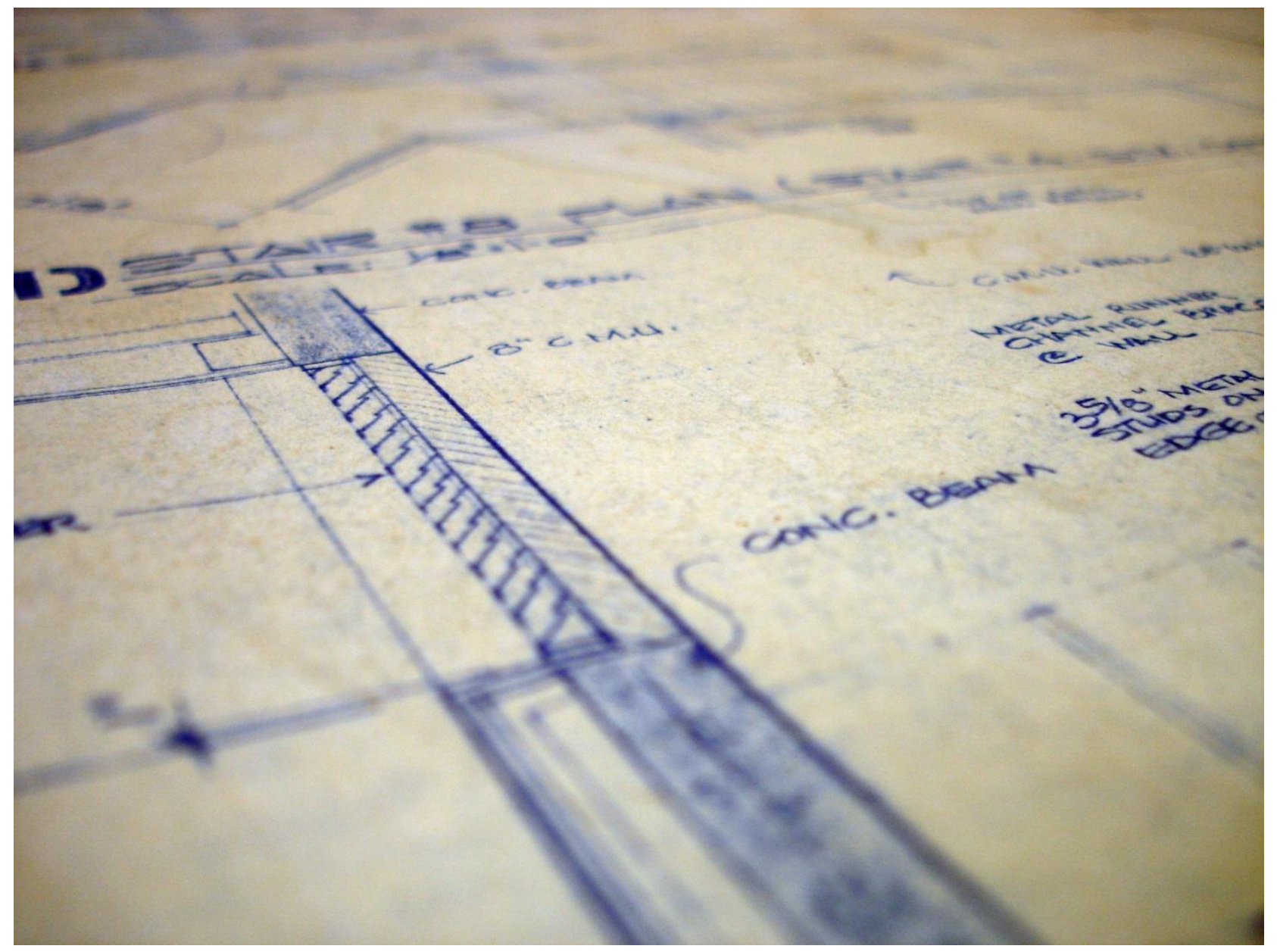




\section{Strategic planning}

- Address Public Services staff concerns

- Enhance Cataloging services

- Share results with Collection Development

- Further research 


\section{Public Services - Concerns}

- Broken links

- OCLC \# missing from records

- Records for print and electronic journals 


\section{Error in public display}

\section{Journal of international relations}

- Journal

Library Has: v.10-v.12

Request this item for pickup.

\begin{tabular}{|l|l|l|}
\hline Location & Call Number & Status \\
\hline Remote Storage (click to request) & Periodical JX V. 11 JL 1920-AP 1921 & AVAlLABLE \\
\hline Remote Storage (click to request) & Periodical JX V. 10 JL 1919-AP 1920 & AVAILABLE \\
\hline Remote Storage (click to request) & Periodical JX V. 12 JL 1921-AP 1922 & AVAlLABLE \\
\hline
\end{tabular}




\section{Edit in staff mode}

b14217363

TITLE Journal of international relations.

CALL NUMBER Periodical JX

LOCATIONS $\mathbf{w}$

LIB. HAS v.10-v.12

Summary

Record

¡14857376

Item-Level Holds

0

Bib-Level Holds
Enter target row (1-3)

Move record(s) to 1

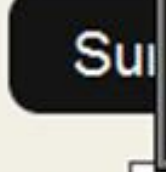

Sul

View

\begin{tabular}{c|c|c|}
\hline \# Record Number \\
\hline
\end{tabular}

\begin{tabular}{cc|c}
\hline & 1 & i14857388 \\
\hline & 2 & i14857376
\end{tabular}

$3 \longdiv { 1 1 4 8 5 7 3 9 x }$

\section{OK Cancel}

Delete

Select

BARCODE 1001574848 1001574847 1001574849
VOLUME V. $11 \mathrm{JL} 1920$-... V. $10 \mathrm{JL} 1919-\ldots$ V. $12 \mathrm{JL} \mathrm{1921-...}$ 


\section{Public display corrected}

\section{Journal of international relations}

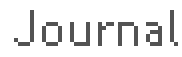

Library Has: v.10-v.12

Request this item for pickup.

\begin{tabular}{|l|l|l|}
\hline Location & Call Number & Status \\
\hline Remote Storage (click to request) & Periodical JX V. 10 JL 1919-AP 1920 & AVAlLABLE \\
\hline Remote Storage (click to request) & Periodical JX V. 11 JL 1920-AP 1921 & AVAILABLE \\
\hline Remote Storage (click to request) & Periodical JX V. 12 JL 1921-AP 1922 & AVAILABLE \\
\hline
\end{tabular}




\section{Future Research}

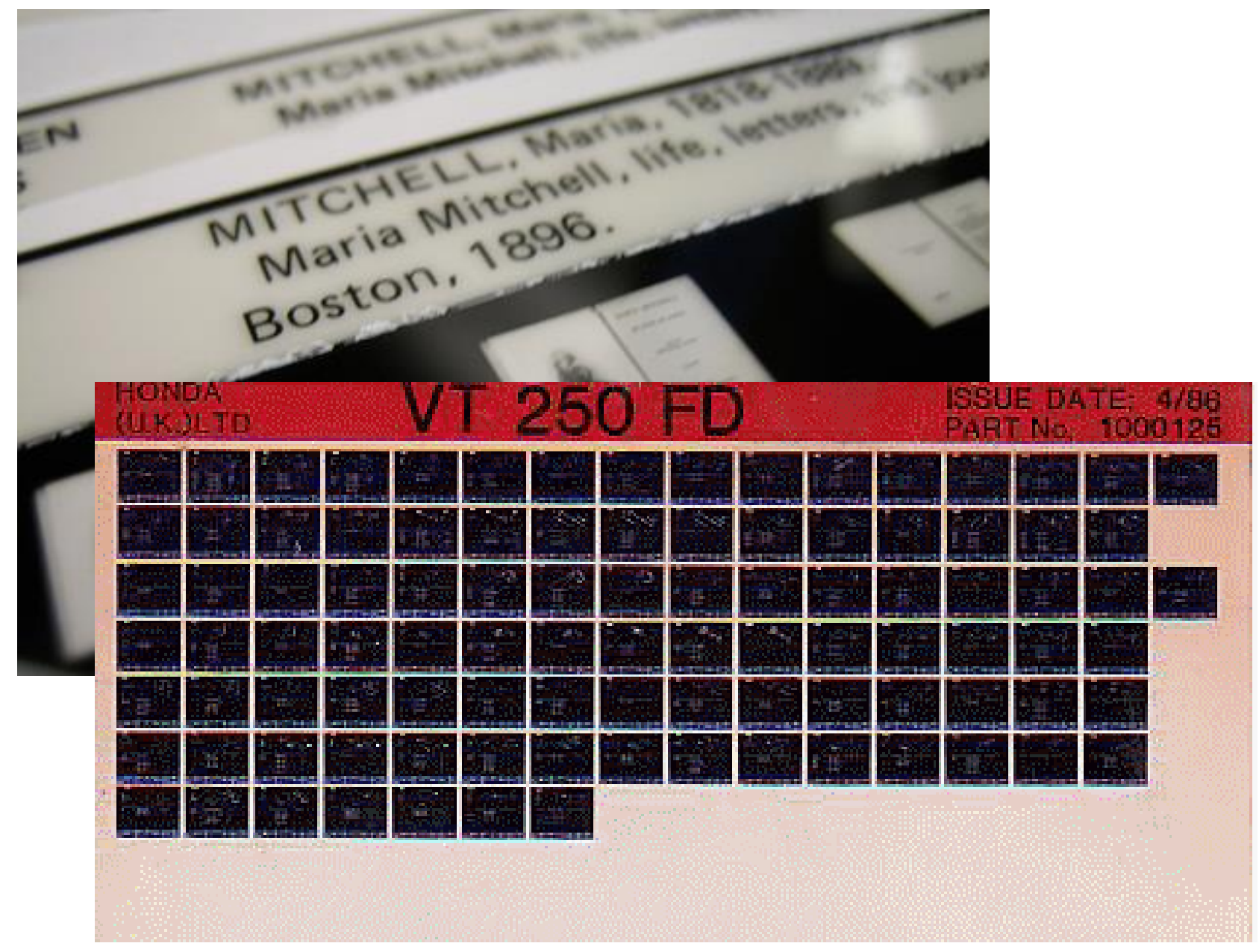


Next: focus groups?

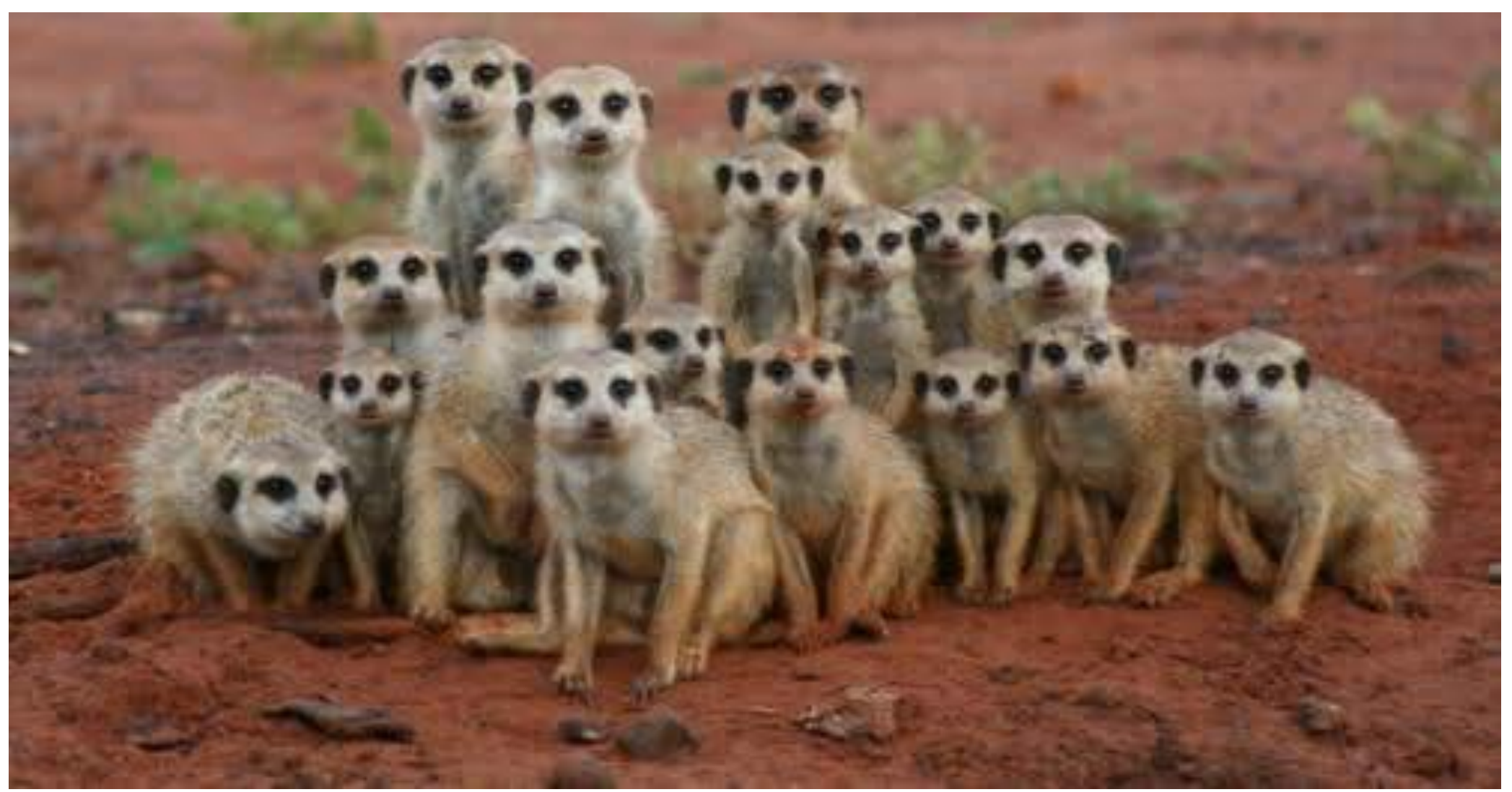




\section{Tips for survey administration}

- Purpose

- Management support

- Feedback

- Timing

- Institutional Review Board (IRB) requirements

- Incentives 


\title{
Further Information
}

\section{Literature Review}

http://tinyurl.com/nn6mdrs

\author{
Survey Instrument
}

http://tinyurl.com/nbdtjpe

\section{Presentation}

Will be available in UNT Digital Library 


\section{Questions?}

A green light to greatness:

Catherine Sassen

Catherine.Sassen@unt.edu

Kathryn Loafman

Kathryn.Loafman@unt.edu

Rebecca Welch

Rebecca.Welch@unt.edu 